\title{
Paramagnetism-based NMR restraints provide maximum allowed probabilities for the different conformations of
}

partially independent protein domains

Ivano Bertini ${ }^{1,2}$, Yogesh K. Gupta ${ }^{1}$, Claudio Luchinat ${ }^{1,3}$, Giacomo Parigi ${ }^{1,3}$, Massimiliano Peana ${ }^{1}$, Luca Sgheri ${ }^{4}$, Jing Yuan $^{1}$

${ }^{1}$ Magnetic Resonance Center (CERM), University of Florence, Via Luigi Sacconi 6, 50019 Sesto

Fiorentino, Italy. ${ }^{2}$ Department of Chemistry, University of Florence, Via della Lastruccia 3, 50019

Sesto Fiorentino, Italy. ${ }^{3}$ Department of Agricultural Biotechnology, University of Florence, via Maragliano 75-77, 50144 Florence, Italy. ${ }^{4}$ Istituto per le Applicazioni del Calcolo-Sezione di Firenze, Polo Scientifico_CNR, Via Madonna del Piano 10, 50019 Sesto Fiorentino, Italy

\section{SUPPORTING INFORMATION}




\section{Preliminary considerations on pcs and $r d c$}

In a multidomain protein containing a paramagnetic ion in one domain, pcs and rdc data on other domains, hereafter indicated as $\delta_{j}, j=1, \ldots, m$, are observables that depend only on the position of these domains, assumed to behave as rigid bodies, in a molecular frame provided by the magnetic susceptibility anisotropy tensor and centered on the paramagnetic ion. The position and orientation of the rigid bodies can be identified by a $(t, R)$ couple, where $t$ is a translation and $R$ a rotation. Suppose the experimental observables $\tilde{\delta}_{j}$ are available: if the rigid body samples different $(t, R)$ couples over time, $\widetilde{\delta}_{j}$ are averages relative to the different positions of the body. It can be easily shown that any set of observables $\left\{\widetilde{\delta}_{j}\right\}$ can be reconstructed using a finite set of $(t, R)$ couples. In other words, a solution always exists with $n \leq m+1$ positions $\left\{\left(t_{i}, R_{i}\right)\right\}$, averaged by non-negative probability values $w_{i}$ (summing up to1), such that

$$
\tilde{\delta}_{j}=\sum_{i=1}^{n} w_{i} \delta_{j}\left(t_{i}, R_{i}\right)
$$

Since $\tilde{\delta}_{j}$ are affected by experimental errors, these must be taken into account. To this end, we should define a target function (TF) representing the error, for instance:

$$
T F\left(w_{i}, t_{i}, R_{i}\right)=\sum_{j=1}^{m}\left|\tilde{\delta}_{j}-\sum_{i=1}^{n} w_{i} \delta_{j}\left(t_{i}, R_{i}\right)\right|^{2}
$$

and carefully select a threshold $\varepsilon$ for the noise level (see below). Then, any set $\left\{\left(w_{i}, t_{i}, R_{i}\right)\right\}$ such that $T F\left(w_{i}, t_{i}, R_{i}\right) \leq \varepsilon$ is considered to be a good reconstruction, and hence a solution. The actual formula may be more complicated, involving different normalization factors.

The mathematical limit $n=m+1$ is however very pessimistic, especially for large $m$, and usually only a fraction of this number of positions is needed to obtain a good agreement between measured and reconstructed data (see ${ }^{1}$ for estimates of this number in the rdc alone case). On the 
other hand, the set of solutions is expected to be very large. A possible approach to explore the conformational space experienced by the system is thus to rank all the obtained solutions according to the $w_{i}$ values of the conformation with largest $w_{i}$ within each solution. The set of conformations with largest $w_{i}$ are likely to contain all the preferred conformations experienced by the system.

\section{The algorithm}

If both pcs and rdc are available, for every fixed conformation $(t, R)$ we are interested in its maximum allowed probability (MAP).

It is utterly impractical to sample the coupled six dimensional space of translations and rotations. Note that in the systems under investigation the different domains are prevented to move away, being attached with a short linker, so the translation must have constrained values. Moreover, a part of the data (the rdc) are not influenced by the translation, so we should avoid the dependence on $t$. We can sum up all the probabilities of the conformations having the same orientation, thus obtaining:

$$
\operatorname{MAP}(R)=\max _{\left\{\left(w_{i}, t_{i}, R_{i}\right\}\right\} \in S}\left\{\Sigma_{k} w_{k}: R_{k}=R\right\}
$$

This definition coincides in the case of the rdc alone with the original one given in ${ }^{1}$. From the numerical point of view the case of two conformations with exactly the same rotational part never happens (and can anyway be prevented using a penalization term). The previous definition can then be recast as:

$$
\operatorname{MAP}(R) \approx \max _{\left\{\left(w_{i}, t_{i}, R_{i}\right\}\right\} \in S}\left\{w_{0}: R_{0}=R, R_{i} \neq R \forall i>0\right\} .
$$

In other words, we fix an orientation $R=R_{0}$ and let the corresponding translation $t_{0}$ vary, together with the remaining orientations $R_{i}$. The maximal weight $w_{0}$ of the orientation $R_{0}$ is then MAP(R).

The proposed favoured orientations $R$ are those where $\operatorname{MAP}(R)$ is large, so we should be looking for rotations $R=\bar{R}$ corresponding to maxima of $\operatorname{MAP}(R)$. However, from the numerical point of view it is much easier to fix a value $w_{0}$ and look for orientations $R=R_{0}$ minimizing 
$T F\left(w_{i}, t_{i}, R_{i}\right)$. The $w_{0}$ value should be chosen according to the following rules: (i) it should be smaller than the maximum of $\operatorname{MAP}(\mathrm{R})$. In this way the minimization of $T F\left(w_{i}, t_{i}, R_{i}\right)$ with respect to $R_{0}$ produces an orientation $\tilde{R}$ such that $T F\left(w_{i}, t_{i}, R_{i}\right) \leq \varepsilon$; (ii) it should be close to the maximal value of $\operatorname{MAP}(R)$. In this way the set of orientations for which $\operatorname{MAP}(R) \geq w_{0}$ will be small, so that $\tilde{R} \approx \bar{R}$.

\section{Calculation of $M A P(R)$ from rdc data}

The $\operatorname{MAP}(R)$ values relative to all orientations of the C-terminal domain with respect to the $\mathrm{N}$ terminal domain were first obtained using the rdc values, through the approach proposed in ${ }^{1}$. In such approach, the exact $\operatorname{MAP}(R)$ values are calculated as boundary points of the convex set of data allowed by the $\chi$ tensors, using a geometrical iterative algorithm based on the vertices selection procedure of the simplex method. The program, previously working only in the case of two tensors, has now been adapted to be used with any number of tensors (i.e., metals).

The calculation of the $\operatorname{MAP}(R)$ value is based on the property that $\operatorname{MAP}(R)$ corresponds to the largest $p$ value for which the quantity $\frac{\bar{\chi}^{k}-p R^{*} \chi^{k} R}{1-p}$ belongs to the set of all possible mean magnetic susceptibility tensors that are compatible with the real $\chi^{k}$ tensors for any metal $k$. This set represents the convex space $V^{k}$, with dimension $5 k . \bar{\chi}^{k}$ indicates the averaged tensors as calculated from the rdc measured for the C-terminal domain. Since $\frac{\bar{\chi}^{k}-p R^{*} \chi^{k} R}{1-p}=\bar{\chi}^{k}-\frac{p}{1-p} \theta$, where $\theta=\bar{\chi}^{k}-R^{*} \chi^{k} R$, the problem reduces to find the $\frac{p}{1-p}$ factor for which the ray $\theta$ (defined in the space with dimension $5 k$ ) starting from the point $\bar{\chi}^{k}$ intersects the boundary of the space $V^{k}$. The actual computation of this unique boundary point can be performed using a geometrical algorithm. We only know some points on the boundary of $V^{k}$, the generating points $T^{*} \chi^{k} T$ for any rotation $T$. 
This algorithm first determines a simplex (i.e. a prism with dimension $5 k$ ) containing $\bar{\chi}^{k}$, and whose $5 k+1$ vertices are generating points. The algorithm then determines the face of this simplex intersecting the ray $\theta$, and the intersection point $P_{1}$. A new generating point, in the half space not containing $\bar{\chi}^{k}$, is then added to this face to form a new simplex. The procedure can be iteratively repeated to find a succession of points $P_{i}$ on $\theta$ approaching the required boundary point.

\section{Calculation of MAP from pcs and rdc data}

A fit was then performed starting from selected orientations $R_{0}$ with largest $\operatorname{MAP}\left(R_{0}\right)$ values, with fixed weight $w_{0}$, to be complemented by other 9 conformations, so that the sum of all weights is 1 . A simulated annealing minimization procedure was applied for the determination of the other 9 conformations, together with the translation $t_{0}$ relative to $R_{0}$. The simulated annealing method was selected because it is able to overcome local minima. In order to ensure that the global minimum of $T F\left(w_{0}\right)$ is actually reached, a simplex minimization was also performed on $R_{0}$. This resulted in an external fit procedure on the orientations with the largest weights.

In the internal simulated annealing minimization, the three Euler angles and the three translation parameters defining the position of the $\mathrm{C}$-terminal domain with respect to the $\mathrm{N}$-terminal domain in each conformation were left free as fit parameters, together with the weight of the conformation. Translations were restrained so that the distance between the last $\mathrm{C}^{\alpha}$ atom of the $\mathrm{N}$ terminal domain $\left(\mathrm{C}^{\alpha}\right.$ of Asp 78) and the first atom of the $\mathrm{C}$-terminal domain $\left(\mathrm{C}^{\alpha}\right.$ of Ser 81$)$ cannot exceed that given by the fully extended conformation of the intervening residues. This minimization results in a very large number of fit parameters, that need to be handled carefully. The most important feature was the addition of a conformation at a time during the minimization process, in order to smoothly adjust the set of conformations providing the best agreement with the experimental restraints. The following protocol was thus developed:

1) a first orientation for the C-terminal domain was fixed, with a fixed weight 
2) a second conformation was randomly added (both as rotation and translation of the C-terminal domain) as starting point for a minimization procedure, where the fit parameters were the translation parameters of the first conformation, and both the rotation and translation parameters of the second conformation.

3) a third conformation was added. The translation parameters of the first conformation with fixed orientation were taken from the result of the fit at the previous step. The translation and rotation of the other two conformations were initially set as the best-fit values obtained for the second conformation in the previous step. Their weight was set to half value of the weight of such conformation. A minimization was then performed on the translation parameters of the first conformation, on the rotation and translation parameters of the second and third conformations, and on their weights.

4) a $n$th conformation was introduced, an so on up to a total of 10 conformations. The rotation and translation parameters of all $n-1$ conformations were initially set according to the results of the minimization procedure at the previous step. The rotation and translation parameters of the $n$th conformation were initially set equal to the rotation and translation parameters of the conformation with largest weight (except the first conformation with fixed orientation) in the previous minimization step, and the weight of that conformation and of the new conformation were set equal to one half of such a value. A minimization was then performed from these starting values on all rotation and translation parameters and on the weights of all conformations, with the exception of the rotation parameters of the first conformation and of its weight.

5) the cycle was stopped after the minimization including 10 conformations. The parameters defining the orientation of the first conformation were left free to change using an external simplextype minimization, in order to obtain the orientation $R_{0}$ with fixed weight $w_{0}$ providing the smallest $T F$. The calculations were then repeated starting from point (1) until convergence was reached. The final target function was used to evaluate the consistency of the weight imposed to the first fixed orientation with the experimental data. 
In the cycle, conformations with negligible weights were continuously removed. In order to improve the speed and reliability of the procedure, the internal simulated annealing minimization to be performed for different $R_{0}$ orientations took advantage of a database of $\left(w_{i}, t_{i}, R_{i}\right)$ providing the lowest target functions in the previous steps, to be used as starting points for the minimization procedure.

The whole cycle was repeated with increasing weight of the first conformation with fixed orientation until the target function exceeded the present $\varepsilon$ value. The largest allowed weight constitutes the maximum allowed probability, MAP, of the selected conformation.

\section{Synthetic tests}

The conformational space experienced by the systems under investigation is quite wide, as indicated by the small rdc values measured on the C-terminal domain with respect to those predicted for the N-terminal domain (see Fig. 2). This indicates that the C-terminal domain assumes a wide range of orientations with respect to the N-terminal domain. In a first synthetic test, the range of orientations was modelled with a probability given by a Gaussian probability distribution in the space of rotations around one conformation of maximal probability. The algorithm for the determination of MAP was thus tested for synthetic data calculated assuming such a model. Rdc and pcs data were first simulated from the average of rdc and pcs obtained for a very large number (50000) of protein conformations generated using such Gaussian probability distribution around one selected conformation. Calculations were performed considering 5 metal ions substituted in the second binding loop of the N-terminal domain, with the same magnetic susceptibility tensors obtained for Tb-, Tm- and Dy-CaM plus other two with similar magnitude, differently oriented

from the former. The variance of the distribution was adjusted so that averaged rdc values were of the same order as the experimental ones. 
Real and averaged anisotropy tensors were then used to calculate $\operatorname{MAP}(R)$. Figure S3 shows the obtained $\operatorname{MAP}(R)$ using 3 or 5 metals. The graph should be read in the following way. A point $(x, y)$ on the graph means that a fraction $x$ of all orientations have a value of $\operatorname{MAP}(R) \leq y$. The $\operatorname{MAP}(R)$ obviously reduces while raising the number of metals. More interesting is the fact that with an increased number of metals the rightmost part of the graph tends to have a steeper slope. This is an indication that the identification is increasingly reliable, because there is a small group of orientations having a significantly higher $\operatorname{MAP}(R)$ value with respect to the others. Data relative to six metals were also generated, and confirmed that there is no benefit in adding a further metal after the fifth, as well known from the theory. ${ }^{1,2}$

Rdc data relative to the 5 average tensors clearly identify the conformation with maximal probability that has been used to generate the data. Figure S4 shows the orientations with largest $\operatorname{MAP}(R)$ values, together with the Gaussian center used to generate the data. Points on the sphere represent the directions of the first helix on the C-terminal domain, with its first residue being placed in the center. The colors (see figure legend) represent the angle between i) the projection of the vector connecting the atoms $C^{\prime}$ of residue Ala 88 and $C^{\alpha}$ of residue Gly 113 , and ii) the projection of the north pole direction, on the plane tangent to the sphere in each point. The intensity of the colors increases with MAP. It is immediately apparent that only one small region has the largest $\operatorname{MAP}(R)$ values, which correctly identifies the privileged orientations (see Fig. S5C). The calculations thus show that, even if data are generated according to a continuous distribution law, such as the Gaussian law, the discrete probability approach may nevertheless be used to obtain meaningful results.

The calculations were repeated with averaged rdc data affected by an error of $\pm 0.5 \mathrm{~Hz}$. Again, the orientations with largest $\operatorname{MAP}(R)$ are in agreement with the maximal probability orientation used to generate the data, indicating the robustness of the approach.

Things are more complicated if only the three metals corresponding to the Tb-, Tm- and Dy$\mathrm{CaM}$ tensors are considered. In this case it is in fact clear that several orientations, grouped in three 
regions, have about the same $\operatorname{MAP}(R)$ values (see Fig. S5A) of 0.35-0.37, and only one of the three contains the correct one. Actually, the program developed to fit both pcs and rdc data clearly indicates that only the conformation depicted in blue in Fig. S5C has smaller target function when its weight is fixed to 0.35 . The contribution of pcs to the target function for increasing weights of the three representative orientations with large $\operatorname{MAP}(R)$ is shown in Figure S5B. Therefore, pcs can be used to complement rdc data obtained using three metal ions to validate and refine the protein conformations with largest MAP values. Again, the calculations were repeated with the averaged rdc data affected by an error of $\pm 0.5 \mathrm{~Hz}$, and with the averaged pcs data affected by an error of $\pm 30 \%$. A good agreement is still obtained between the conformation with maximal probability used to generate the data and the conformation with largest MAP. The TF of the conformations outside the correct region remains $20 \%$ larger than the $\mathrm{TF}$ of the correct conformation.

In a second synthetic test, pcs and rdc data were generated for three metals using a probability distribution resulting from the sum of two Gaussian functions centered on two different orientations. Again, the $\operatorname{MAP}(R)$ values calculated from the rdc data were able to recover these two orientations together with other ghosts (see Fig. S6). The latter were successfully removed by the pcs, so that the two correct conformations with maximal probability were recovered.

\section{Preparation of $A S$}

Plasmid pDEST-17 (Invitrogen) able to express his-tagged AS was transformed into BL-21AI E. coli strain. A primary culture was from a single bacterial colony diluted up to 1 L either with the LB media or M9 minimal media supplemented with the appropriate isotopically labeled nitrogen $\left(1.2 \mathrm{~g}{ }^{15} \mathrm{NH}_{4} \mathrm{Cl} / \mathrm{L}\right)$ and carbon sources $\left(3 \mathrm{~g}{ }^{13} \mathrm{C}\right.$-glucose/L), and grown in a shaker. AS expression was induced at mid-log phase stage with $0.2 \%$ L-arabinose. The bacterial cells were harvested at $7 \mathrm{hrs}$ of post-arabinose induction, centrifuged and resuspended in $15 \mathrm{~mL}$ of ice-chilled lysis buffer (0.05 M Tris, $0.5 \mathrm{M} \mathrm{NaCl}, 0.01 \mathrm{M}$ Imidazole, $\mathrm{pH}$ 7.2) containing $0.05 \mathrm{mg} / \mathrm{mL}$ lysozyme and $1 \mathrm{mM}$ PMSF and incubated on ice for $30 \mathrm{~min}$ to achieve the maximum activity of lysozyme. A 
lysate obtained by sonication was boiled for $15 \mathrm{~min}$ to inactivate all the bacterial proteases coexpressed with his-tagged AS, and centrifuged. The supernatant containing his-tagged AS was loaded onto a hi-trap chelating column charged with a $\mathrm{NiSO}_{4}$ solution. Other proteins were removed from the column by passing $25 \mathrm{~mL}$ of lysis buffer followed by $25 \mathrm{~mL}$ of wash buffer containing 0.02 M imidazole. The his-tagged AS was then eluted with $15 \mathrm{~mL}$ of elution buffer, containing 0.3 M imidazole. The eluate fractions were pooled and their buffer was exchanged with the factor Xa protease buffer (0.02 M Tris, $\left.0.1 \mathrm{M} \mathrm{NaCl}, 0.002 \mathrm{M} \mathrm{CaCl}_{2}, \mathrm{pH} 8.0\right)$. The His tag was cleaved off from native AS by incubating the uncut protein with the factor Xa protease enzyme for 5 hrs at 310 K. Finally, the native un-tagged AS was purified by size exclusion chromatography using a Superdex 75 column (Amersham pharmacia). The purity of the protein was checked by 1\% SDS$15 \%$ PAGE electrophoresis.

Reference List

(1) Longinetti, M.; Luchinat, C.; Parigi, G.; Sgheri, L. Inv.Probl. 2006, 22, 1485-1502.

(2) Tolman, J. R. J.Am.Chem.Soc. 2002, 124, 12020-12030. 


\section{Figures}

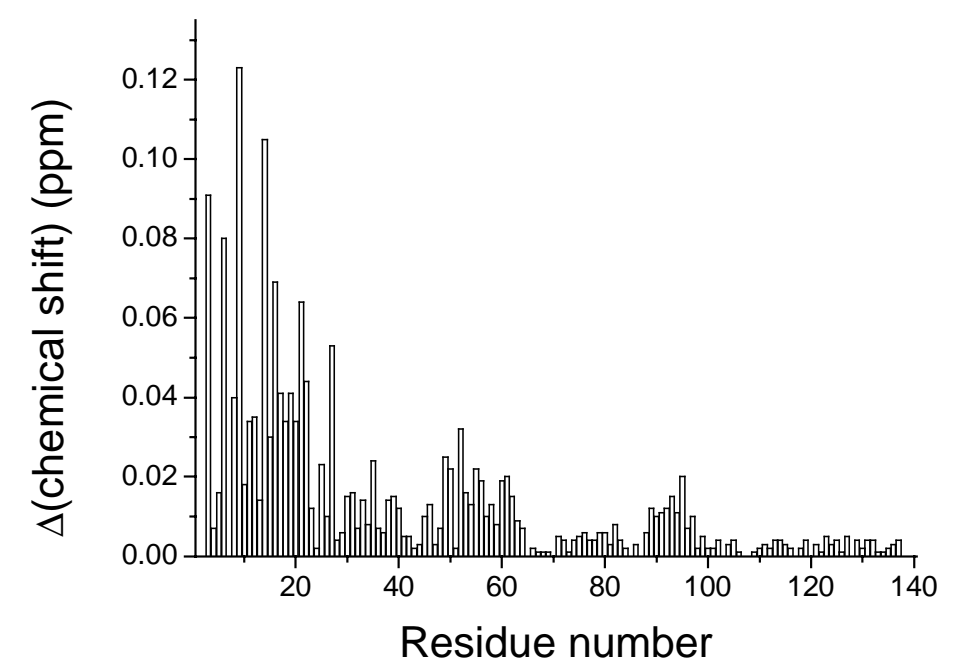

Figure S1. Plot of the change in chemical shift observed for the AS HN and $\mathrm{N}$ backbone atoms in the $1: 1{ }^{15} \mathrm{~N}$ AS-CaM complex. Shifts are reported as a weighted average using the formula $\Delta \delta=\left[\left(\Delta \delta_{\mathrm{H}}\right)^{2}+\left(\Delta \delta_{\mathrm{N}} \times\left(\gamma_{\mathrm{N}} / \gamma_{\mathrm{H}}\right)\right)^{2}\right]^{0.5}$. 

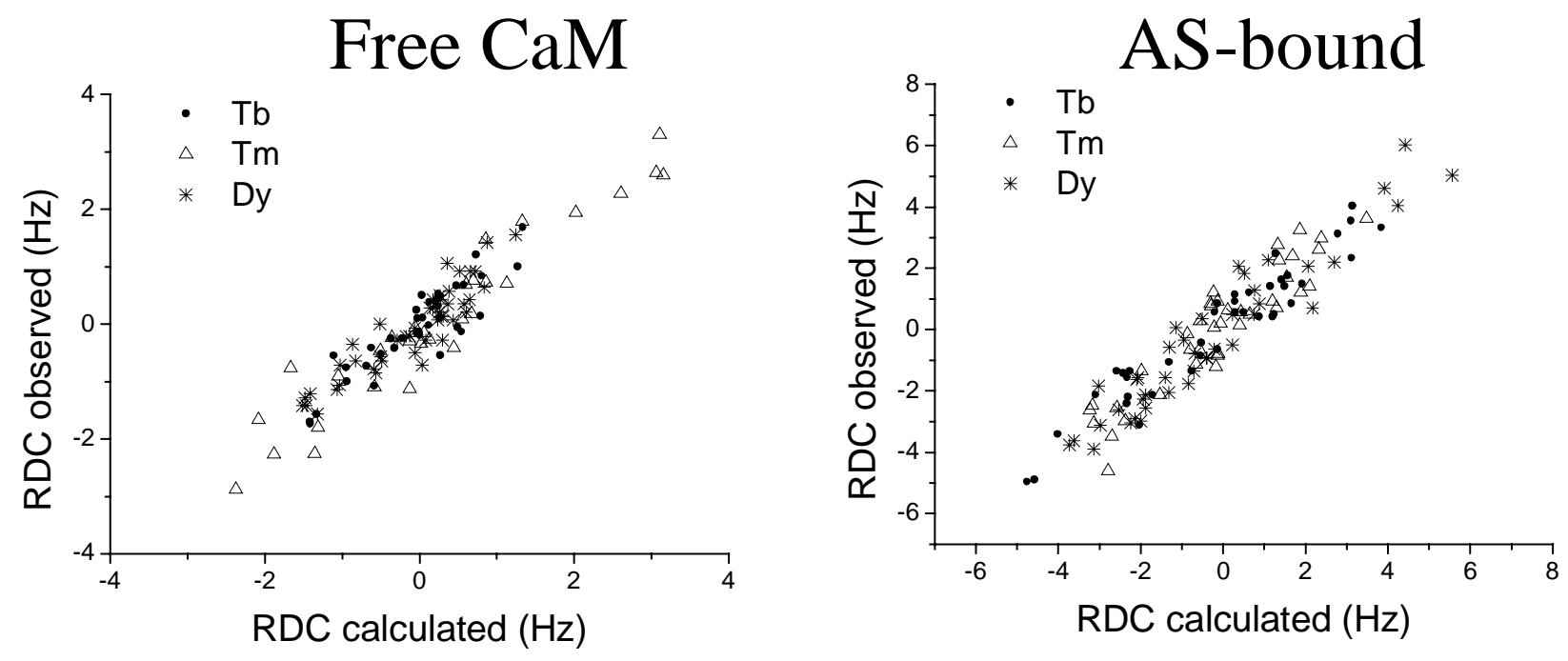

Figure S2. Observed versus calculated values of rdc of C-terminal HN for the terbium(III), thulium(III) and dysprosium(III) CaM derivatives in the presence of AS. 


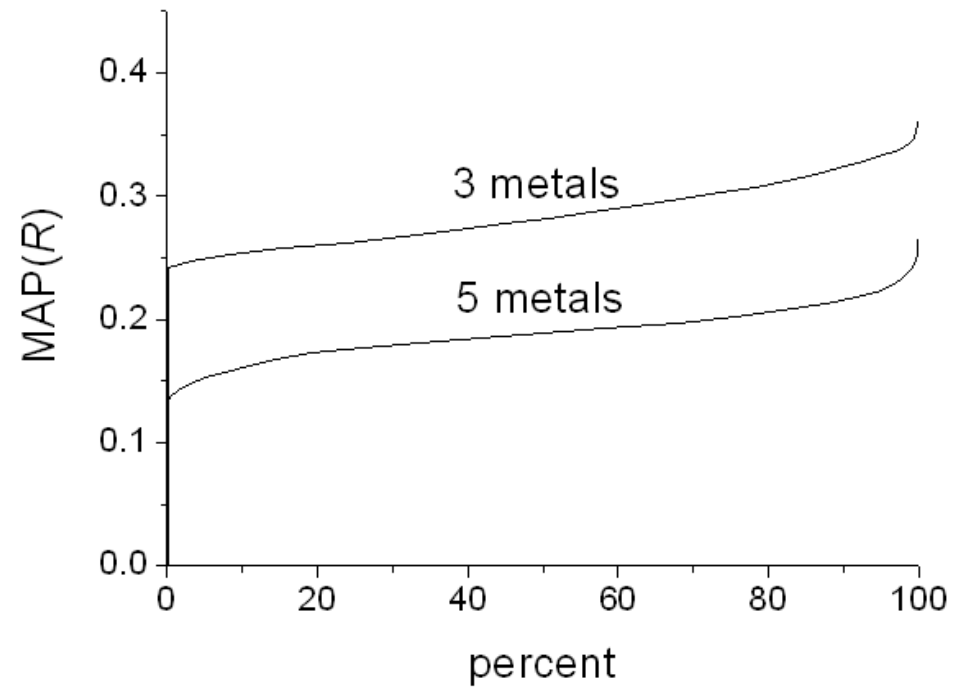

Figure S3. Calculated $\operatorname{MAP}(R)$ values for simulated data using 3 or 5 metals. A point $(x, y)$ on the graph means that $x \%$ of all orientations has a value of $\operatorname{MAP}(R) \leq y$. 

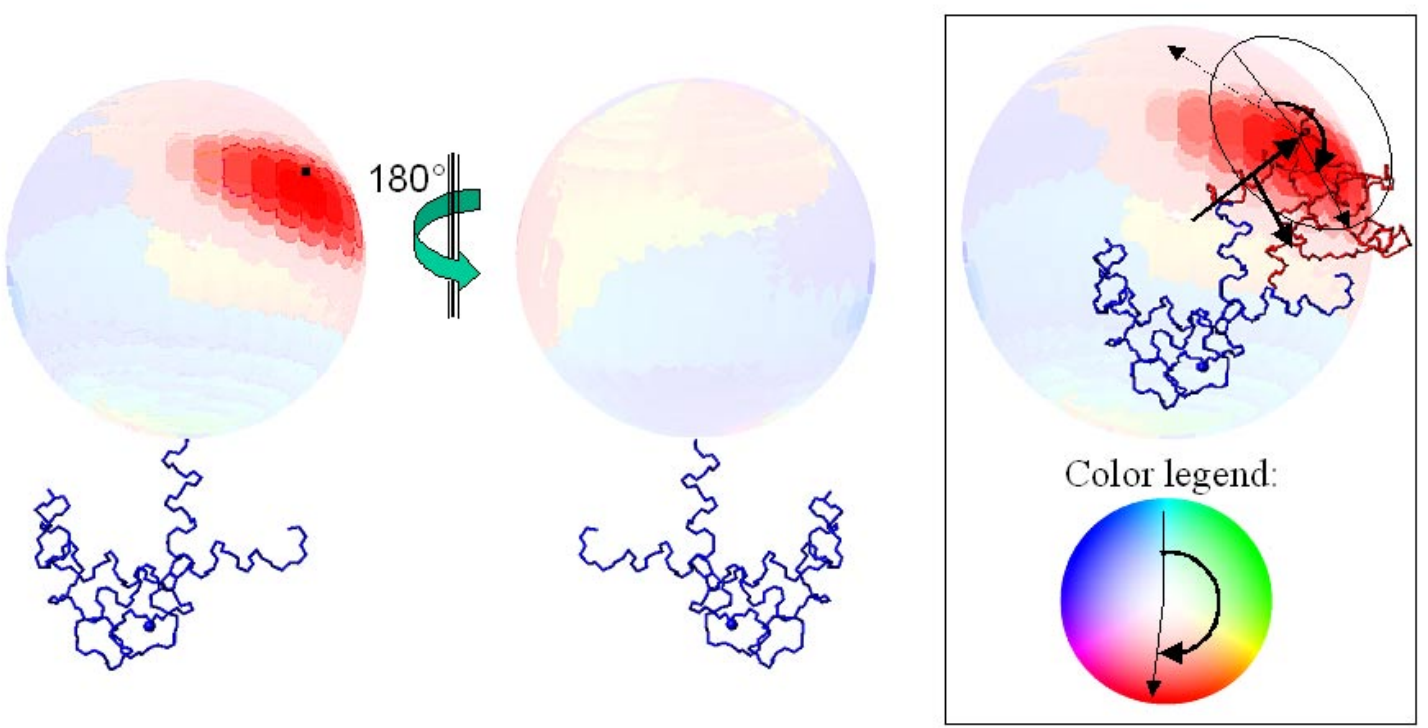

Figure S4. $\operatorname{MAP}(R)$ values calculated using simulated rdc data from 5 metals for all relative orientations of the $\mathrm{C}$-terminal domain with respect to the $\mathrm{N}$-terminal domain. The points on the sphere represent the directions of the first helix on the C-terminal domain, with its first residue being placed in the center. The colors represent the angle between the projections of the vector connecting the atoms $C^{\prime}$ of residue Ala 88 and $C^{\alpha}$ of residue Gly 113 (roughly perpendicular to the axis of the first helix of the C-terminal domain) and the projection of the north pole direction, on the plane tangent to the sphere in each point, according to the legend. The intensity of the color increases with increasing $\operatorname{MAP}(R)$. The Gaussian centre used to generate the data (black dot) is also indicated. 
A

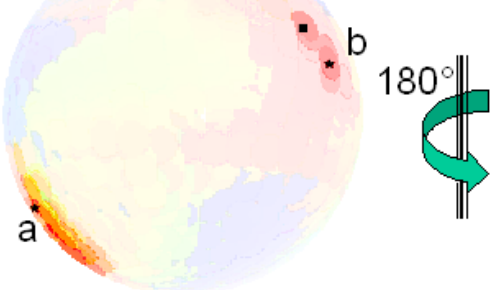

$\mathrm{B}$

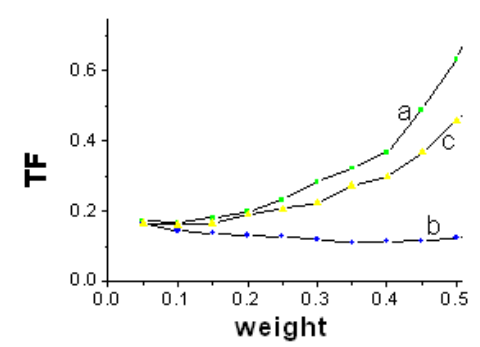

C

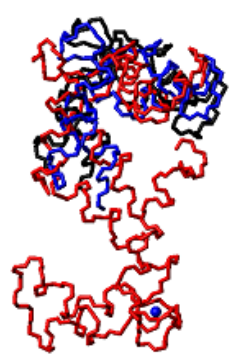

$\mathrm{D}$

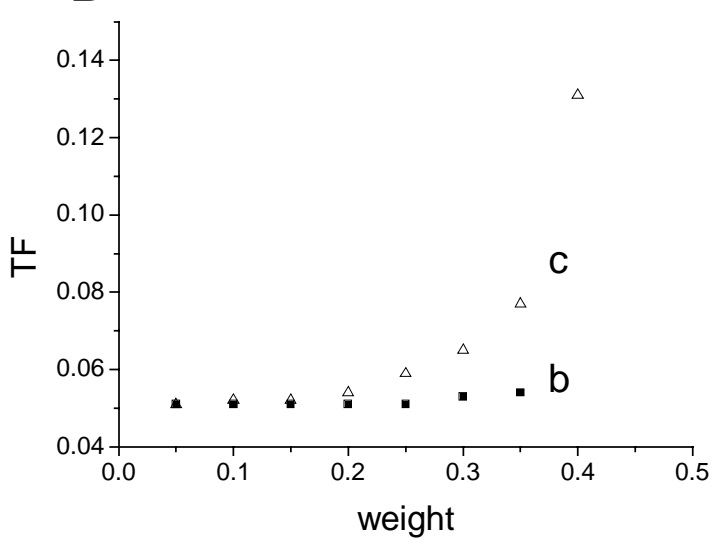

Figure S5. (A) $\operatorname{MAP}(R)$ values calculated using simulated rdc data from 3 metals for all relative orientations of the $\mathrm{C}$-terminal domain with respect to the $\mathrm{N}$-terminal domain (see caption to Fig. S4). (B) Pseudocontact shift contribution (not normalized) to the target function for increasing weights of the fixed conformation among the four with largest $\operatorname{MAP}(R)$. The smaller target function relative to one conformation clearly select it as the correct one. (C) Protein preferred conformations used to generate the data (red), and obtained from the fit using 5 (black) or 3 (blue) metals. (D) Target function (from both pcs and rdc) calculated for increasing weights of conformations $\mathrm{b}$ and $\mathrm{c}$, using average rdc data affected by an error of $\pm 0.5 \mathrm{~Hz}$, and averaged pcs data affected by an error of $\pm 30 \%$. 

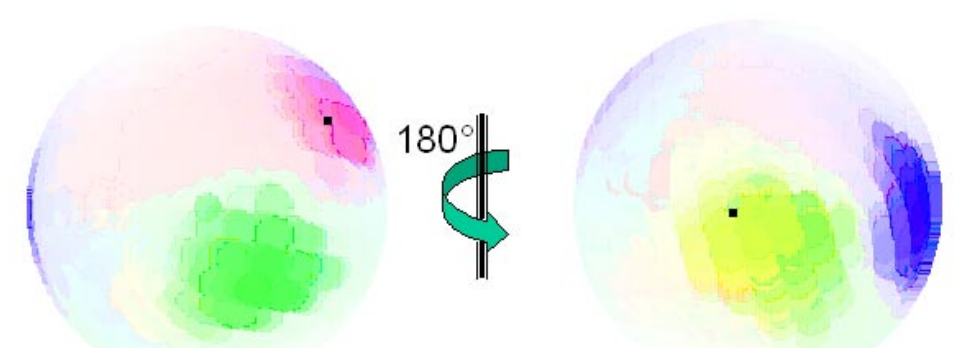

Figure S6. $\operatorname{MAP}(R)$ values calculated using simulated rdc data from 3 metals for all relative orientations of the C-terminal domain with respect to the $\mathrm{N}$-terminal domain (see caption to Fig. S4). The two Gaussian centres used to generate the data (black dots) are also indicated. 
A

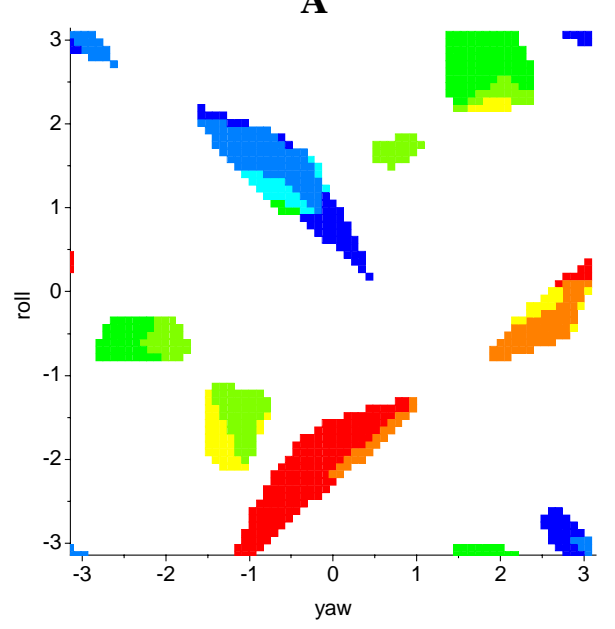

B

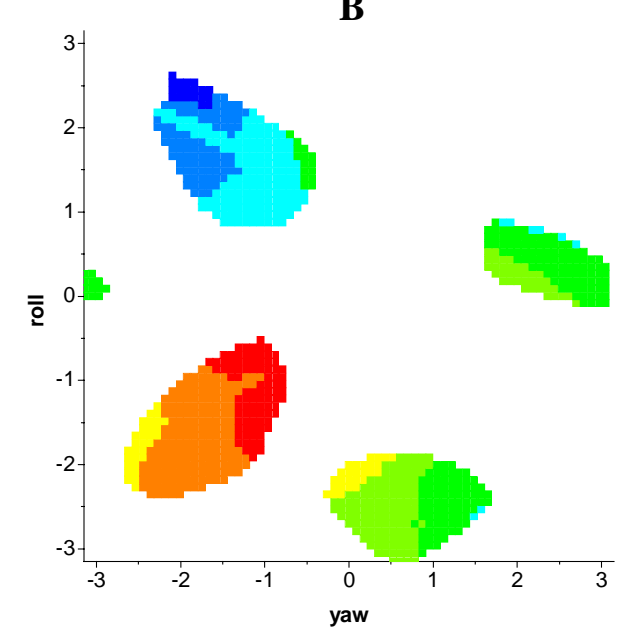

C

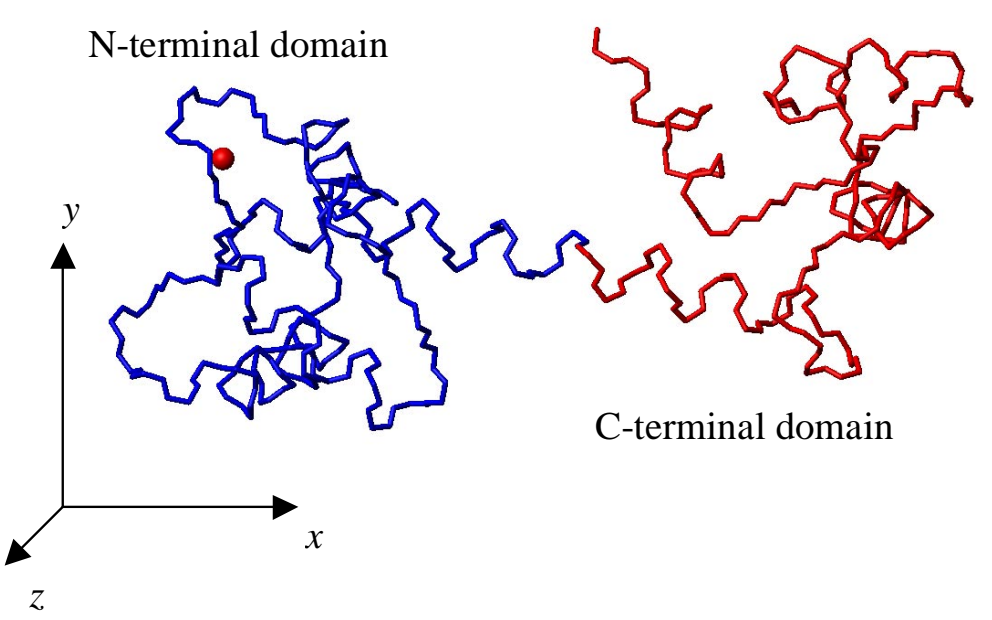

Figure S7. Euler angles of the rotations of the C-terminal domain corresponding to the conformations with largest $\mathrm{MAP}(R)$ values for free $\mathrm{CaM}$ (A) and CaM-AS (B). The color corresponds to the pitch angle according to the following color map: red is $\pi / 2$, green is 0 and blue is $-\pi / 2$. The conformation corresponding to zero rotation is shown in $\mathrm{C}$. 
Table S1. Polypeptide backbone N, HN Chemical Shift for Human Calmodulin and Human Calmodulin complexed with alpha Synuclein at pH 6.8, $200 \mathrm{mM} \mathrm{KCl}, 20 \mathrm{mM}$ MES at $298 \mathrm{~K}$

\begin{tabular}{|c|c|c|c|c|c|}
\hline CAM & $\mathbf{N}$ & HN & CAM-SYN & $\mathbf{N}$ & HN \\
\hline A1 & 124.073 & 9.142 & A1 & 124.153 & 9.065 \\
\hline D2 & 116.728 & 7.999 & D2 & 116.870 & 8.005 \\
\hline Q3 & 119.818 & 8.247 & Q3 & 119.777 & 8.211 \\
\hline L4 & 121.408 & 7.719 & L4 & 121.503 & 7.740 \\
\hline T5 & 113.107 & 8.729 & T5 & 112.905 & 8.725 \\
\hline E6 & 120.528 & 9.001 & E6 & 120.527 & 8.999 \\
\hline E7 & 119.557 & 8.709 & E7 & 119.555 & 8.708 \\
\hline Q8 & 120.422 & 7.726 & Q8 & 120.444 & 7.707 \\
\hline I9 & 119.651 & 8.400 & I9 & 119.525 & 8.362 \\
\hline A10 & 121.252 & 7.969 & A10 & 121.281 & 7.969 \\
\hline E11 & 119.794 & 7.754 & E11 & 119.855 & 7.741 \\
\hline F12 & 120.253 & 8.551 & F12 & 119.981 & 8.525 \\
\hline K13 & 123.728 & 9.228 & K13 & 123.649 & 9.232 \\
\hline E14 & 120.422 & 7.726 & E14 & 120.521 & 7.727 \\
\hline A15 & 122.736 & 7.928 & A15 & 122.542 & 7.953 \\
\hline F16 & 119.411 & 8.836 & F16 & 119.254 & 8.808 \\
\hline S17 & 112.780 & 7.907 & S17 & 113.109 & 7.989 \\
\hline L18 & 120.797 & 7.360 & L18 & 121.024 & 7.346 \\
\hline F19 & 115.188 & 7.258 & F19 & 114.578 & 7.177 \\
\hline D20 & 117.656 & 7.793 & D20 & 117.392 & 7.768 \\
\hline K21 & 124.489 & 7.639 & K21 & 124.356 & 7.628 \\
\hline D22 & 113.928 & 8.032 & D22 & 114.053 & 8.048 \\
\hline G23 & 109.313 & 7.658 & G23 & 109.367 & 7.653 \\
\hline D24 & 120.764 & 8.337 & D24 & 120.898 & 8.388 \\
\hline G25 & 113.348 & 10.576 & G25 & 113.121 & 10.537 \\
\hline T26 & 111.485 & 8.076 & T26 & 111.540 & 8.119 \\
\hline I27 & 126.587 & 9.812 & I27 & 126.730 & 9.861 \\
\hline T28 & 116.431 & 8.442 & $\mathrm{~T} 28$ & 116.409 & 8.403 \\
\hline T29 & 112.805 & 9.134 & T29 & 112.782 & 9.200 \\
\hline K30 & 120.945 & 7.637 & K30 & 121.062 & 7.617 \\
\hline E31 & 121.510 & 7.581 & E31 & 121.602 & 7.609 \\
\hline L32 & 120.812 & 8.728 & L32 & 120.698 & 8.712 \\
\hline G33 & 105.618 & 8.627 & G33 & 105.880 & 8.688 \\
\hline T34 & 117.693 & 7.877 & T34 & 117.786 & 7.897 \\
\hline V35 & 122.139 & 7.611 & V35 & 122.228 & 7.667 \\
\hline M36 & 118.576 & 8.446 & M36 & 118.578 & 8.411 \\
\hline R37 & 119.018 & 8.518 & R37 & 118.825 & 8.521 \\
\hline S38 & 118.950 & 7.882 & S38 & 119.007 & 7.889 \\
\hline L39 & 120.797 & 7.360 & L39 & 120.717 & 7.335 \\
\hline G40 & 107.028 & 7.845 & G40 & 107.042 & 7.829 \\
\hline Q41 & 118.573 & 7.758 & Q41 & 118.409 & 7.761 \\
\hline $\mathrm{N} 42$ & 116.336 & 8.695 & $\mathrm{~N} 42$ & 116.456 & 8.704 \\
\hline P43 & & & P43 & & \\
\hline T44 & 112.806 & 8.757 & T44 & 112.905 & 8.725 \\
\hline E45 & 120.810 & 8.776 & E45 & 120.805 & 8.773 \\
\hline A46 & 120.806 & 8.248 & A46 & 120.803 & 8.240 \\
\hline E47 & 118.703 & 7.671 & E47 & 118.704 & 7.660 \\
\hline L48 & 120.642 & 8.139 & L48 & 120.252 & 8.105 \\
\hline Q49 & 118.236 & 8.181 & Q49 & 118.323 & 8.201 \\
\hline
\end{tabular}




\begin{tabular}{|c|c|c|c|c|c|}
\hline D50 & 120.118 & 8.083 & D50 & 120.210 & 8.098 \\
\hline M51 & 119.567 & 7.824 & M51 & 119.362 & 7.834 \\
\hline I52 & 118.284 & 7.666 & I52 & 118.243 & 7.700 \\
\hline N53 & 117.661 & 8.553 & N53 & 117.907 & 8.587 \\
\hline E54 & 116.202 & 7.506 & E54 & 116.048 & 7.430 \\
\hline V55 & 108.112 & 7.181 & V55 & 109.685 & 7.204 \\
\hline D56 & 121.807 & 7.640 & D56 & 121.376 & 7.673 \\
\hline A57 & 131.937 & 8.589 & A57 & 131.651 & 8.434 \\
\hline D58 & 114.351 & 8.256 & D58 & 114.641 & 8.300 \\
\hline G59 & 108.624 & 7.546 & G59 & 108.642 & 7.549 \\
\hline D60 & 120.818 & 8.269 & D60 & 120.855 & 8.286 \\
\hline G61 & 113.207 & 10.386 & G61 & 113.014 & 10.327 \\
\hline T62 & 111.815 & 8.231 & T62 & 111.736 & 8.237 \\
\hline I63 & 122.812 & 8.927 & I63 & 122.627 & 8.819 \\
\hline D64 & 128.845 & 8.882 & D64 & 128.978 & 8.931 \\
\hline F65 & 117.725 & 8.716 & F65 & 117.669 & 8.723 \\
\hline P66 & & & P66 & & \\
\hline E67 & 118.811 & 8.098 & E67 & 118.798 & 8.121 \\
\hline F68 & 124.048 & 8.968 & F68 & 124.048 & 8.917 \\
\hline L69 & 118.990 & 8.367 & L69 & 118.503 & 8.320 \\
\hline $\mathrm{T} 70$ & 115.569 & 7.529 & T70 & 115.540 & 7.702 \\
\hline M71 & 121.866 & 7.766 & M71 & 121.484 & 7.754 \\
\hline M72 & 116.662 & 8.077 & M72 & 116.854 & 8.009 \\
\hline A73 & 122.523 & 8.242 & A73 & 121.964 & 8.255 \\
\hline R74 & 116.838 & 7.582 & R74 & 117.104 & 7.494 \\
\hline K75 & 118.703 & 7.671 & K75 & 118.704 & 7.660 \\
\hline M76 & 118.625 & 7.828 & M76 & 118.364 & 7.772 \\
\hline K77 & 120.650 & 7.844 & K77 & 120.472 & 7.776 \\
\hline D78 & 121.770 & 8.271 & D78 & 121.436 & 8.214 \\
\hline T79 & 114.552 & 8.062 & T79 & 114.250 & 7.999 \\
\hline D80 & 123.232 & 8.402 & D80 & 123.329 & 8.380 \\
\hline S81 & 117.286 & 8.396 & S81 & 117.357 & 8.424 \\
\hline E82 & 122.637 & 8.444 & E82 & 122.453 & 8.420 \\
\hline E83 & 119.826 & 8.247 & E83 & 119.755 & 8.219 \\
\hline E84 & 118.846 & 8.152 & E84 & 118.703 & 8.144 \\
\hline I85 & 122.213 & 7.974 & I85 & 121.892 & 7.942 \\
\hline R86 & 121.913 & 8.368 & R86 & 121.806 & 8.380 \\
\hline E87 & 118.834 & 8.099 & E87 & 118.809 & 8.116 \\
\hline A88 & 122.162 & 7.910 & A88 & 121.969 & 7.938 \\
\hline F89 & 119.076 & 8.510 & F89 & 118.913 & 8.507 \\
\hline R90 & 115.766 & 7.642 & R90 & 115.858 & 7.684 \\
\hline V91 & 118.415 & 7.536 & V91 & 118.292 & 7.488 \\
\hline F92 & 116.202 & 7.506 & F92 & 116.056 & 7.433 \\
\hline D93 & 117.143 & 7.816 & D93 & 116.765 & 7.816 \\
\hline K94 & 126.023 & 7.672 & K94 & 126.026 & 7.689 \\
\hline D95 & 114.043 & 8.144 & D95 & 114.063 & 8.160 \\
\hline G96 & 109.391 & 7.749 & G96 & 109.398 & 7.761 \\
\hline N97 & 119.535 & 8.273 & N97 & 119.605 & 8.297 \\
\hline G98 & 113.092 & 10.614 & G98 & 113.037 & 10.617 \\
\hline Y99 & 115.858 & 7.568 & Y99 & 115.973 & 7.584 \\
\hline I100 & 127.312 & 10.094 & I100 & 127.296 & 10.099 \\
\hline S101 & 123.862 & 8.915 & S101 & 123.862 & 8.903 \\
\hline A102 & 122.946 & 9.149 & A102 & 123.079 & 9.168 \\
\hline A103 & 118.418 & 8.214 & A103 & 118.469 & 8.227 \\
\hline E104 & 119.663 & 7.820 & E104 & 119.846 & 7.845 \\
\hline
\end{tabular}




\begin{tabular}{|c|c|c|c|c|c|}
\hline L105 & 121.336 & 8.586 & L105 & 121.061 & 8.519 \\
\hline R106 & 117.595 & 8.524 & R106 & 117.753 & 8.575 \\
\hline H1 07 & 118.596 & 7.877 & H1 07 & 118.399 & 7.915 \\
\hline V108 & 118.541 & 7.925 & V108 & 118.409 & 7.919 \\
\hline M109 & 116.756 & 8.200 & M109 & 116.295 & 8.203 \\
\hline T110 & 114.895 & 8.116 & T110 & 115.221 & 8.166 \\
\hline N111 & 122.159 & 7.900 & N111 & 122.278 & 7.853 \\
\hline L112 & 118.781 & 7.836 & L112 & 119.019 & 7.892 \\
\hline G113 & 107.028 & 7.845 & G113 & 107.042 & 7.829 \\
\hline E114 & 120.385 & 7.866 & E114 & 120.269 & 7.868 \\
\hline K115 & 123.766 & 8.537 & K115 & 124.023 & 8.561 \\
\hline L116 & 124.721 & 8.067 & L116 & 124.919 & 8.057 \\
\hline T117 & 114.651 & 9.179 & T117 & 114.752 & 9.215 \\
\hline D118 & 121.102 & 8.865 & D118 & 121.100 & 8.867 \\
\hline E119 & 119.146 & 8.651 & E119 & 119.136 & 8.648 \\
\hline E120 & 120.422 & 7.726 & E120 & 120.521 & 7.727 \\
\hline V121 & 121.378 & 8.071 & V121 & 121.088 & 8.033 \\
\hline D122 & 119.837 & 8.023 & D122 & 119.702 & 8.034 \\
\hline E123 & 119.688 & 7.938 & E123 & 119.698 & 7.960 \\
\hline M124 & 119.748 & 7.765 & M124 & 119.834 & 7.744 \\
\hline I125 & 118.548 & 7.923 & I125 & 118.298 & 7.895 \\
\hline R126 & 118.437 & 8.121 & R126 & 118.437 & 8.144 \\
\hline E127 & 115.950 & 7.909 & E127 & 116.044 & 7.868 \\
\hline A128 & 118.967 & 7.315 & A128 & 118.849 & 7.294 \\
\hline D129 & 117.579 & 7.816 & D129 & 117.700 & 7.835 \\
\hline I130 & 127.728 & 8.333 & I130 & 127.720 & 8.298 \\
\hline D131 & 116.643 & 8.256 & D131 & 116.638 & 8.279 \\
\hline G132 & 109.055 & 7.528 & G132 & 109.230 & 7.580 \\
\hline D133 & 120.801 & 8.285 & D133 & 121.073 & 8.313 \\
\hline G134 & 113.043 & 10.340 & G134 & 112.942 & 10.316 \\
\hline Q135 & 115.483 & 7.912 & Q135 & 115.468 & 7.917 \\
\hline V136 & 125.449 & 9.095 & V136 & 125.446 & 9.092 \\
\hline N137 & 128.979 & 9.458 & N137 & 129.076 & 9.480 \\
\hline Y138 & 118.646 & 8.452 & Y138 & 118.612 & 8.415 \\
\hline E139 & 118.425 & 8.091 & E139 & 118.486 & 8.087 \\
\hline E140 & 119.831 & 8.724 & E140 & 119.882 & 8.728 \\
\hline F141 & 124.916 & 8.957 & F141 & 124.846 & 8.894 \\
\hline V142 & 119.479 & 8.478 & V142 & 119.300 & 8.524 \\
\hline Q143 & 117.997 & 7.295 & Q143 & 118.191 & 7.377 \\
\hline M144 & 119.274 & 7.881 & M144 & 119.039 & 7.828 \\
\hline M145 & 114.769 & 7.806 & M145 & 114.799 & 7.743 \\
\hline T146 & 112.143 & 7.531 & T146 & 111.570 & 7.521 \\
\hline A147 & 126.718 & 7.749 & A147 & 126.923 & 7.755 \\
\hline K148 & 125.732 & 7.689 & K148 & 125.886 & 7.759 \\
\hline
\end{tabular}


Table S2. ${ }^{1} \mathrm{H}$ resonance assignment for Human N60D Calmodulin complexed with alpha Synuclein at $\mathrm{pH}$ 6.8, $200 \mathrm{mM} \mathrm{KCl}, 20 \mathrm{mM} \mathrm{MES}$ at $298 \mathrm{~K}$

\begin{tabular}{|c|c|c|c|c|c|c|c|c|c|c|c|}
\hline Residue & HA & & HB2 & HB3 & HG2 & HG3 & & HD2 & HD3 & HE2 & HE3 \\
\hline A1 & 4.080 & & QB 1.314 & & & & & & & & \\
\hline D2 & 4.445 & & QB 2.646 & & & & & & & & \\
\hline Q3 & 4.333 & & 1.948 & 2.152 & QG 2.304 & & & & & & \\
\hline L4 & 4.622 & & 1.447 & 1.761 & HG 1.744 & & & QD1 0.860 & QD2 0.894 & & \\
\hline T5 & 4.403 & & HB 4.739 & & & & QG2 1.283 & & & & \\
\hline E6 & 3.928 & & QB 2.005 & & QG 2.346 & & & & & & \\
\hline E7 & 4.013 & & QB 1.889 & & 2.249 & 2.289 & & & & & \\
\hline Q8 & 3.794 & & 2.168 & 1.615 & QG 2.333 & & & & & & \\
\hline 19 & 3.646 & & HB 1.896 & & HG12 1.035 & HG13 1.776 & QG2 1.070 & QD1 0.818 & & & \\
\hline A10 & 4.077 & & QB 1.471 & & & & & & & & \\
\hline E11 & 4.085 & & 1.975 & 2.059 & 1.848 & 2.313 & & & & & \\
\hline F12 & 4.985 & & QB 3.422 & & & & & QD 7.128 & & QE 7.191 & \\
\hline K13 & 3.956 & & QB 1.867 & & 1.004 & 1.129 & & 1.168 & 1.296 & 2.303 & 2.539 \\
\hline E14 & 4.011 & & 1.993 & 2.211 & QG 2.404 & & & & & & \\
\hline A15 & 4.229 & & QB 1.909 & & & & & & & & \\
\hline F16 & 3.203 & & 2.870 & 3.121 & & & & QD 6.562 & & QE 6.990 & \\
\hline S17 & 4.053 & & QB 3.969 & & & & & & & & \\
\hline L18 & 3.931 & & 1.513 & 1.681 & HG 1.493 & & & QD1 0.807 & QD2 0.656 & & \\
\hline F19 & 4.189 & & QB 2.607 & & & & & & & & \\
\hline D20 & 4.490 & & 1.432 & 2.278 & & & & & & & \\
\hline K21 & 3.906 & & QB 1.822 & & 1.412 & 1.479 & & QD 1.639 & & QE 2.956 & \\
\hline D22 & 4.536 & & 2.585 & 3.004 & & & & & & & \\
\hline G23 & 3.771 & 3.838 & & & & & & & & & \\
\hline D24 & 4.450 & & 2.409 & 3.009 & & & & & & & \\
\hline G25 & 3.636 & 4.350 & & & & & & & & & \\
\hline T26 & 5.211 & & HB 3.879 & & & & QG2 1.010 & & & & \\
\hline $\mathbf{I 2 7}$ & 4.849 & & HB 1.805 & & & QG1 1.165 & QG2 0.813 & QD1 0.247 & & & \\
\hline T28 & 4.768 & & HB 4.736 & & & & QG2 1.255 & & & & \\
\hline T29 & 3.737 & & HB 4.155 & & & & QG2 1.224 & & & & \\
\hline K30 & 4.097 & & 1.756 & 1.835 & & 1.450 & & QD 1.610 & & & \\
\hline E31 & 4.011 & & QB 2.318 & & QG 2.440 & & & & & & \\
\hline L32 & 4.050 & & 1.465 & 1.832 & HG 1.755 & & & QD1 0.813 & QD2 0.768 & & \\
\hline G33 & 3.928 & 3.513 & & & & & & & & & \\
\hline T34 & 3.860 & & HB 4.266 & & & & QG2 1.225 & & & & \\
\hline V35 & 3.487 & & HB 1.925 & & & QG1 1.165 & QG2 0.650 & & & & \\
\hline M36 & 4.041 & & 1.998 & 1.817 & 2.561 & 2.629 & & & & & \\
\hline R37 & 4.743 & & QB 1.853 & & QG 1.789 & & & 3.075 & 3.269 & & \\
\hline S38 & 4.351 & & 4.056 & 3.998 & & & & & & & \\
\hline L39 & 4.445 & & QB 1.829 & & HG 1.758 & & & QD1 0.783 & QD2 0.7991 & & \\
\hline G40 & 4.221 & 3.761 & & & & & & & & & \\
\hline Q41 & 4.440 & & 1.603 & 2.044 & QG 2.188 & & & & & & \\
\hline $\mathrm{N} 42$ & 5.163 & & 2.457 & 2.733 & & & & HD21 6.707 & HD22 7.513 & & \\
\hline $\mathbf{P 4 3}$ & 4.696 & & 1.895 & 2.186 & QG 1.892 & & & 3.558 & 3.243 & & \\
\hline T44 & 4.404 & & HB 4.703 & & & & QG2 1.301 & & & & \\
\hline E45 & 3.948 & & QB 2.003 & & QG 2.296 & & & & & & \\
\hline $\mathrm{A} 46$ & 4.054 & & QB 1.337 & & & & & & & & \\
\hline E47 & 3.980 & & 1.862 & 2.017 & QG 2.281 & & & & & & \\
\hline L48 & 4.028 & & 1.218 & 2.036 & HG 1.719 & & & QD1 0.811 & QD2 0.798 & & \\
\hline Q49 & 3.771 & & 2.086 & 2.153 & 2.358 & 2.438 & & & & & \\
\hline
\end{tabular}




\begin{tabular}{|c|c|c|c|c|c|c|c|c|c|c|c|}
\hline D50 & 4.360 & & 2.629 & 2.749 & & & & & & & \\
\hline M51 & 3.994 & & 1.948 & 2.246 & 2.490 & 2.729 & & & & & \\
\hline $\mathbf{I 5 2}$ & 3.447 & & HB 1.860 & & HG12 0.922 & HG13 1.660 & QG2 0.675 & QD1 0.6111 & & & \\
\hline N53 & 4.334 & & 2.818 & 2.928 & & & & HD21 6.904 & HD22 7.832 & & \\
\hline E54 & 3.990 & & 1.976 & 2.073 & 2.230 & 2.398 & & & & & \\
\hline V55 & 4.399 & & HB 2.301 & & & QG1 0.770 & QG2 0.861 & & & & \\
\hline D56 & 4.512 & & 2.562 & 2.674 & & & & & & & \\
\hline A57 & 4.217 & & QB 1.482 & & & & & & & & \\
\hline D58 & 4.549 & & 2.604 & 2.981 & & & & & & & \\
\hline G59 & 3.716 & 3.863 & & & & & & & & & \\
\hline D60 & 4.439 & & 2.436 & 2.903 & & & & & & & \\
\hline G61 & 4.139 & 3.423 & & & & & & & & & \\
\hline T62 & 4.861 & & HB 3.850 & & & & QG2 0.985 & & & & \\
\hline I63 & 5.190 & & HB 1.961 & & HG12 0.849 & HG13 1.600 & QG2 1.202 & QD1 0.772 & & & \\
\hline D64 & 5.330 & & 3.110 & 2.851 & & & & & & & \\
\hline F65 & 3.933 & & 2.010 & 2.749 & & & & QD 6.676 & & QE 7.146 & \\
\hline P66 & 3.793 & & QB 1.868 & & QG 1.851 & & & 3.649 & 3.750 & & \\
\hline E67 & 3.584 & & 2.040 & 2.527 & 2.182 & 2.327 & & & & & \\
\hline F68 & 3.913 & & 3.459 & 3.175 & & & & QD 6.902 & & QE 7.124 & \\
\hline L69 & 3.306 & & 1.540 & 1.077 & HG 0.942 & & & QD1 0.580 & & & \\
\hline T70 & 3.693 & & HB 4.269 & & & & QG2 1.146 & & & & \\
\hline M71 & 3.708 & & QB 1.915 & & QG 2.113 & & & & & & \\
\hline M72 & 3.929 & & QB 1.651 & & QG 1.818 & & & & & & \\
\hline A73 & 4.044 & & QB 1.332 & & & & & & & & \\
\hline R74 & 4.031 & & QB 1.779 & & QG 1.588 & & & QD 3.050 & & & \\
\hline K75 & 4.136 & & QB 1.84 & & QG 1.399 & & & QD 1.608 & & QE 2.836 & \\
\hline M76 & 4.325 & & 2.016 & 2.124 & 2.548 & 2.656 & & & & & \\
\hline K77 & 4.258 & & 1.755 & 1.844 & QG 1.420 & & & QD 1.627 & & QE 2.942 & \\
\hline D78 & 4.684 & & 2.611 & 2.713 & & & & & & & \\
\hline T79 & 4.257 & & HB 4.206 & & & & QG2 1.165 & & & & \\
\hline D80 & 4.662 & & 2.604 & 2.709 & & & & & & & \\
\hline S81 & 4.395 & & 3.918 & 3.984 & & & & & & & \\
\hline E82 & 4.093 & & QB 2.072 & & QG 2.308 & & & & & & \\
\hline E83 & 3.991 & & QB 2.077 & & QG 2.297 & & & & & & \\
\hline E84 & 3.998 & & QB 2.062 & & QG 2.281 & & & & & & \\
\hline I85 & 3.981 & & HB 2.117 & & HG 2.010 & & QG2 1.074 & QD1 0.744 & & & \\
\hline R86 & 4.095 & & 1.635 & 2.014 & 1.500 & 1.831 & & QD 2.919 & & & \\
\hline E87 & 4.101 & & 2.034 & 2.144 & QG 2.323 & & & & & & \\
\hline A88 & 4.168 & & QB 1.898 & & & & & & & & \\
\hline F89 & 3.064 & & 2.945 & 3.168 & & & & QD 6.560 & & QE 7.164 & \\
\hline R90 & 3.814 & & QB 1.893 & & 1.632 & 1.710 & & QD 3.169 & & & \\
\hline V91 & 3.439 & & HB 2.102 & & & QG1 0.963 & QG2 0.620 & & & & \\
\hline F92 & 4.168 & & QB 2.621 & & & & & QD 7.271 & & & \\
\hline D93 & 4.488 & & 1.307 & 2.264 & & & & & & & \\
\hline K94 & 4.088 & & QB 1.820 & & 1.381 & 1.449 & & QD 1.615 & & QE 2.939 & \\
\hline D95 & 4.518 & & 2.607 & 3.024 & & & & & & & \\
\hline G96 & 3.752 & 3.853 & & & & & & & & & \\
\hline N97 & 4.586 & & 2.602 & 3.361 & & & & HD21 7.541 & HD22 8.038 & & \\
\hline G98 & 3.998 & 3.373 & & & & & & & & & \\
\hline Y99 & 4.996 & & 2.486 & 2.416 & & & & QD 6.675 & & QE 6.908 & \\
\hline $\mathbf{I 1 0 0}$ & 4.779 & & HB 1.809 & & HG12 0.249 & HG13 1.178 & QG2 0.890 & QD1 0.246 & & & \\
\hline S101 & 4.790 & & 3.934 & 4.364 & & & & & & & \\
\hline $\mathbf{A 1 0 2}$ & 3.865 & & QB 1.422 & & & & & & & & \\
\hline A103 & 3.976 & & QB 1.365 & & & & & & & & \\
\hline
\end{tabular}




\begin{tabular}{|c|c|c|c|c|c|c|c|c|c|c|}
\hline E104 & 4.019 & & 1.910 & 1.981 & QG 2.468 & & & & & \\
\hline L105 & 4.053 & & 1.487 & 1.829 & HG 1.626 & & & QD1 0.813 & QD2 0.938 & \\
\hline R106 & 3.783 & & QB 1.897 & & QG 1.607 & & & 3.076 & 3.180 & \\
\hline H107 & 4.278 & & 3.214 & 3.272 & & & & QD 6.946 & & \\
\hline V108 & 3.460 & & HB 1.931 & & & QG1 0.781 & QG2 0.368 & & & \\
\hline M109 & 4.284 & & 1.929 & 2.107 & 2.568 & 2.659 & & & & \\
\hline T110 & 4.131 & & HB 4.250 & & & & QG2 1.179 & & & \\
\hline N111 & 4.442 & & 2.747 & 2.635 & & & & HD21 7.189 & HD22 7.501 & \\
\hline L112 & 4.324 & & QB 1.759 & & HG 1.790 & & & QD1 0.813 & QD2 0.778 & \\
\hline G113 & 3.760 & 4.182 & & & & & & & & \\
\hline E114 & 4.362 & & 1.677 & 1.916 & 2.061 & 2.193 & & & & \\
\hline K115 & 4.335 & & 1.635 & 1.752 & 1.276 & 1.345 & & & & QE 2.943 \\
\hline L116 & 4.732 & & 1.420 & 1.519 & HG 1.505 & & & QD1 0.744 & QD2 0.771 & \\
\hline T117 & 4.416 & & HB 4.699 & & & & QG2 1.293 & & & \\
\hline D118 & 4.153 & & 2.529 & 2.688 & & & & & & \\
\hline E119 & 4.001 & & 1.868 & 1.980 & QG 2.294 & & & & & \\
\hline E120 & 3.956 & & 1.867 & 2.343 & QG 2.210 & & & & & \\
\hline V121 & 3.557 & & HB 2.164 & & & QG1 0.913 & QG2 0.960 & & & \\
\hline D122 & 4.284 & & 2.577 & 2.734 & & & & & & \\
\hline E123 & 3.982 & & QB 2.010 & & QG 2.300 & & & & & \\
\hline M124 & 3.985 & & 1.980 & 2.182 & QG 2.725 & & & & & \\
\hline I125 & 3.447 & & HB 2.066 & & HG12 1.474 & HG13 1.230 & QG2 0.941 & QD1 0.669 & & \\
\hline R126 & 3.965 & & 1.886 & 2.005 & 1.579 & 1.709 & & QD 3.171 & & \\
\hline E127 & 3.974 & & QB 2.012 & & QG 2.267 & & & & & \\
\hline A128 & 4.349 & & QB 1.378 & & & & & & & \\
\hline D129 & 4.448 & & QB 2.450 & & & & & & & \\
\hline I130 & 3.858 & & HB 1.948 & & HG12 1.284 & HG13 1.641 & QG2 0.8872 & QD1 0.828 & & \\
\hline D131 & 4.501 & & 2.600 & 3.012 & & & & & & \\
\hline G132 & 3.927 & 3.757 & & & & & & & & \\
\hline D133 & 4.440 & & 2.447 & 2.901 & & & & & & \\
\hline G134 & 3.990 & 3.372 & & & & & & & & \\
\hline Q135 & 4.805 & & 1.655 & 1.936 & QG 1.900 & & & & & \\
\hline V136 & 5.168 & & HB 2.256 & & & QG1 1.224 & QG2 0.863 & & & \\
\hline N137 & 5.181 & & 3.192 & 3.252 & & & & HD21 6.708 & HD22 6.961 & \\
\hline Y138 & 3.372 & & 2.336 & 2.021 & & & & QD 6.246 & & QE 6.466 \\
\hline E139 & 3.593 & & 1.919 & 2.047 & QG 2.322 & & & & & \\
\hline E140 & 3.993 & & 2.186 & 2.533 & QG 2.366 & & & & & \\
\hline F141 & 3.908 & & 3.138 & 3.326 & & & & QD 6.890 & & QE 7.119 \\
\hline V142 & 3.071 & & HB 1.820 & & & QG1 0.459 & QG2 0.692 & & & \\
\hline Q143 & 3.829 & & QB 2.084 & & QG 2.355 & & & & & \\
\hline M144 & 4.132 & & QB 2.035 & & QG 2.169 & & & & & \\
\hline M145 & 4.257 & & 1.599 & 2.089 & 2.315 & 2.479 & & & & \\
\hline T146 & 4.237 & & HB 4.211 & & & & QG2 1.135 & & & \\
\hline A147 & 4.242 & & QB 1.343 & & & & & & & \\
\hline K148 & 4.268 & & QB 1.768 & & QG 1.409 & & & QD 1.631 & & QE 2.555 \\
\hline
\end{tabular}


Table S3. ${ }^{13} \mathrm{C}$ resonance assignment for Human N60D Calmodulin complexed with alpha Synuclein at pH 6.8, $200 \mathrm{mM} \mathrm{KCl}, 20 \mathrm{mM}$ MES at $298 \mathrm{~K}$

\begin{tabular}{|c|c|c|c|c|c|c|c|c|c|}
\hline Residue & CA & CB & CG & CG1 & CG2 & CD & CD1 & CD2 & CE \\
\hline A1 & 53.39 & 18.58 & & & & & & & \\
\hline D2 & 55.12 & 40.78 & & & & & & & \\
\hline Q3 & 54.86 & 29.66 & 34.01 & & & & & & \\
\hline L4 & 54.21 & 43.36 & 26.19 & & & & 23.60 & & \\
\hline T5 & 60.58 & 71.14 & & & 21.63 & & & & \\
\hline E6 & 59.97 & 29.03 & 36.51 & & & & & & \\
\hline E7 & 59.74 & 29.31 & 36.47 & & & & & & \\
\hline Q8 & 58.51 & 28.81 & 34.83 & & & & & & \\
\hline I9 & 66.17 & 37.76 & & 30.20 & 17.25 & & 12.83 & & \\
\hline A10 & 55.33 & 17.68 & & & & & & & \\
\hline E11 & 58.88 & 29.17 & 36.02 & & & & & & \\
\hline F12 & 59.05 & 37.67 & & & & & & & \\
\hline K13 & 59.93 & 31.60 & 24.94 & & & 28.60 & & & \\
\hline E14 & 59.17 & 28.81 & 35.93 & & & & & & \\
\hline A15 & 55.11 & 18.02 & & & & & & & \\
\hline F16 & 61.87 & 39.51 & & & & & & & \\
\hline S17 & 61.27 & 62.98 & & & & & & & \\
\hline L18 & 57.03 & 41.36 & & & & & 26.15 & 23.80 & \\
\hline F19 & 59.20 & 40.35 & & & & & & & \\
\hline D20 & 52.27 & 38.73 & & & & & & & \\
\hline K21 & 58.36 & 32.73 & 24.29 & & & 28.78 & & & 41.90 \\
\hline D22 & 52.82 & 39.59 & & & & & & & \\
\hline G23 & 47.22 & & & & & & & & \\
\hline D24 & 53.57 & 40.20 & & & & & & & \\
\hline G25 & 45.37 & & & & & & & & \\
\hline T26 & 59.43 & 72.59 & & & & & & & \\
\hline $\mathbf{I 2 7}$ & 60.31 & 39.48 & & 26.60 & 17.77 & & 13.33 & & \\
\hline T28 & 59.66 & 72.13 & & & 21.80 & & & & \\
\hline T29 & 66.34 & 67.72 & & & 23.22 & & & & \\
\hline K30 & 59.01 & 32.47 & 24.65 & & & 29.17 & & & \\
\hline E31 & 59.09 & 29.45 & 38.39 & & & & & & \\
\hline L32 & 57.98 & 42.42 & 26.00 & & & & 25.80 & 23.30 & \\
\hline G33 & 48.21 & & & & & & & & \\
\hline T34 & 66.69 & 68.43 & & & 21.48 & & & & \\
\hline V35 & 66.03 & 31.19 & & 20.48 & 22.73 & & & & \\
\hline M36 & 58.90 & 30.83 & 32.64 & & & & & & \\
\hline R37 & 59.00 & 29.53 & 29.41 & & & 43.35 & & & \\
\hline S38 & 61.53 & 62.86 & & & & & & & \\
\hline L39 & 54.21 & 42.17 & 26.00 & & & & 22.58 & 25.39 & \\
\hline G40 & 45.44 & & & & & & & & \\
\hline Q41 & 54.27 & 30.36 & 33.75 & & & & & & \\
\hline $\mathrm{N} 42$ & 51.19 & 39.06 & & & & & & & \\
\hline P43 & 62.19 & 31.81 & 27.57 & & & 49.69 & & & \\
\hline T44 & 60.40 & 71.08 & & & 21.73 & & & & \\
\hline
\end{tabular}




\begin{tabular}{|c|c|c|c|c|c|c|c|c|c|}
\hline E45 & 59.75 & 29.25 & 36.30 & & & & & & \\
\hline A46 & 54.88 & 18.01 & & & & & & & \\
\hline E47 & 59.06 & 29.51 & 37.19 & & & & & & \\
\hline L48 & 57.94 & 42.28 & 26.37 & & & & 25.58 & 23.01 & \\
\hline Q49 & 58.58 & 28.20 & 34.45 & & & & & & \\
\hline D50 & 57.33 & 40.02 & & & & & & & \\
\hline M51 & 59.18 & 33.08 & 32.66 & & & & & & \\
\hline I52 & 65.08 & 37.08 & & 28.82 & 15.76 & & 15.86 & & \\
\hline N53 & 55.77 & 38.02 & & & & & & & \\
\hline E54 & 58.89 & 30.19 & 35.94 & & & & & & \\
\hline V55 & 60.68 & 32.79 & & 22.18 & 22.22 & & & & \\
\hline D56 & 53.69 & 40.21 & & & & & & & \\
\hline A57 & 53.81 & 19.53 & & & & & & & \\
\hline D58 & 52.54 & 38.71 & & & & & & & \\
\hline G59 & 46.89 & & & & & & & & \\
\hline D60 & 53.79 & 40.49 & & & & & & & \\
\hline G61 & 45.55 & & & & & & & & \\
\hline T62 & 59.82 & 72.03 & & & 22.12 & & & & \\
\hline I63 & 59.80 & 39.99 & & 27.29 & 18.26 & & 13.78 & & \\
\hline D64 & 51.92 & 41.25 & & & & & & & \\
\hline F65 & 63.19 & 35.75 & & & & & & & \\
\hline P66 & 66.29 & 30.65 & 28.49 & & & 48.78 & & & \\
\hline E67 & 60.04 & 29.44 & & & & & & & \\
\hline F68 & 61.08 & 39.77 & & & & & & & \\
\hline L69 & 57.63 & 40.78 & & & & & 23.96 & & \\
\hline T70 & 66.32 & 68.48 & & & 21.83 & & & & \\
\hline M71 & 58.70 & 29.36 & & & & & & & \\
\hline M72 & 55.68 & & 33.42 & & & & & & \\
\hline A73 & 54.32 & 18.12 & & & & & & & \\
\hline R74 & 58.20 & 30.12 & 26.96 & & & 42.97 & & & \\
\hline K75 & 57.01 & 32.30 & 24.64 & & & & & & 42.01 \\
\hline M76 & 56.38 & 32.75 & 32.28 & & & & & & \\
\hline K77 & 56.37 & 32.76 & 24.60 & & & 29.19 & & & 41.81 \\
\hline D78 & 54.47 & 40.80 & & & & & & & \\
\hline T79 & 61.32 & 69.42 & & & 21.16 & & & & \\
\hline D80 & 54.51 & 41.27 & & & & & & & \\
\hline S81 & 59.08 & 63.68 & & & & & & & \\
\hline E82 & 58.19 & 29.49 & 36.15 & & & & & & \\
\hline E83 & 59.26 & 29.44 & 38.19 & & & & & & \\
\hline E84 & 59.25 & 29.25 & 35.80 & & & & & & \\
\hline I85 & 64.21 & 37.08 & & 29.13 & 18.88 & & 13.09 & & \\
\hline R86 & 59.84 & 29.76 & & & & 42.79 & & & \\
\hline E87 & 59.05 & 28.93 & & & & & & & \\
\hline A88 & 55.32 & 18.07 & & & & & & & \\
\hline F89 & 61.87 & 39.16 & & & & & & & \\
\hline R90 & 58.69 & 30.52 & 27.74 & & & 43.27 & & & \\
\hline V91 & 65.06 & 31.36 & & 22.61 & 21.05 & & & & \\
\hline F92 & 58.71 & 40.62 & & & & & & & \\
\hline D93 & 52.09 & 38.33 & & & & & & & \\
\hline
\end{tabular}




\begin{tabular}{|c|c|c|c|c|c|c|c|c|c|}
\hline K94 & 58.56 & 32.26 & 24.98 & & & 28.78 & & & 41.86 \\
\hline D95 & 53.13 & 39.57 & & & & & & & \\
\hline G96 & 47.03 & & & & & & & & \\
\hline N97 & 52.64 & 38.14 & & & & & & & \\
\hline G98 & 45.03 & & & & & & & & \\
\hline Y99 & 55.94 & 43.00 & & & & & & & \\
\hline I100 & 60.03 & 38.55 & & 26.11 & 17.69 & & 15.18 & & \\
\hline S101 & 55.72 & 66.36 & & & & & & & \\
\hline $\mathbf{A 1 0 2}$ & 55.96 & 18.26 & & & & & & & \\
\hline A103 & 55.12 & 18.28 & & & & & & & \\
\hline E104 & 58.66 & 29.14 & 37.68 & & & & & & \\
\hline L105 & 57.87 & 41.93 & 27.01 & & & & 25.33 & & \\
\hline R106 & 59.49 & 30.29 & 27.30 & & & 43.61 & & & \\
\hline H107 & 59.29 & 29.84 & & & & & & & \\
\hline V108 & 66.20 & 31.70 & & 22.99 & 20.51 & & & & \\
\hline M109 & 57.30 & 31.54 & 33.04 & & & & & & \\
\hline T110 & 65.64 & 68.78 & & & 21.21 & & & & \\
\hline N111 & 55.28 & 38.08 & & & & & & & \\
\hline L112 & 55.31 & 41.92 & 26.13 & & & & & 22.55 & \\
\hline G113 & 45.42 & & & & & & & & \\
\hline E114 & 55.14 & 30.24 & 34.98 & & & & & & \\
\hline K115 & 55.56 & 31.55 & 24.23 & & & & & & \\
\hline L116 & 54.05 & 44.76 & & & & & 26.99 & 23.45 & \\
\hline T117 & 60.67 & 70.96 & & & 21.54 & & & & \\
\hline D118 & 57.76 & 39.69 & & & & & & & \\
\hline E119 & 60.03 & 28.81 & 36.62 & & & & & & \\
\hline E120 & 59.13 & 29.08 & 37.80 & & & & & & \\
\hline V121 & 66.78 & 31.29 & & 23.74 & 21.67 & & & & \\
\hline D122 & 57.45 & 40.21 & & & & & & & \\
\hline E123 & 58.81 & 29.07 & 35.64 & & & & & & \\
\hline M124 & 59.41 & 28.78 & 32.96 & & & & & & \\
\hline I125 & 63.58 & 36.35 & & 28.08 & & & 10.83 & & \\
\hline R126 & 60.27 & 29.86 & 27.46 & & & 43.42 & & & \\
\hline E127 & 58.52 & 29.72 & & & & & & & \\
\hline A128 & 52.11 & 21.16 & & & & & & & \\
\hline D129 & 53.78 & 40.08 & & & & & & & \\
\hline I130 & 63.28 & 38.36 & & 27.76 & 16.95 & & 12.24 & & \\
\hline D131 & 53.69 & 39.67 & & & & & & & \\
\hline G132 & 47.42 & & & & & & & & \\
\hline D133 & 53.78 & 39.85 & & & & & & & \\
\hline G134 & 45.52 & & & & & & & & \\
\hline Q135 & 53.04 & 32.08 & 32.89 & & & & & & \\
\hline V136 & 61.61 & 33.77 & & 22.01 & 22.47 & & & & \\
\hline N137 & 50.97 & 38.31 & & & & & & & \\
\hline Y138 & 62.63 & 37.53 & & & & & & & \\
\hline E139 & 60.16 & 28.53 & 37.19 & & & & & & \\
\hline E140 & 58.33 & 29.73 & 37.58 & & & & & & \\
\hline F141 & 61.28 & 39.60 & & & & & & & \\
\hline V142 & 66.96 & 31.37 & & 22.74 & 21.09 & & & & \\
\hline
\end{tabular}




\begin{tabular}{|l|l|l|l|l|l|l|l|l|l|}
\hline Q143 & 58.64 & 28.17 & 33.76 & & & & & & \\
\hline M144 & 57.66 & 33.15 & & & & & & & \\
\hline M145 & 55.39 & 31.84 & 32.05 & & & & & & \\
\hline T146 & 61.79 & 69.63 & & & 21.14 & & & & \\
\hline A147 & 52.48 & 18.79 & & & & & & & \\
\hline K148 & 56.94 & 32.27 & 24.35 & & & 28.94 & & & 41.98 \\
\hline
\end{tabular}


Table S4. Polypeptide backbone N, HN Chemical Shift for apo Human Synuclein and apo Human alpha Synuclein complexed with Calmodulin at pH 6.8, $200 \mathrm{mM} \mathrm{KCl,} 20 \mathrm{mM}$ MES at $298 \mathrm{~K}$

\begin{tabular}{|c|c|c|c|c|c|}
\hline Syn & $\mathbf{N}$ & HN & SynCalm & $\mathbf{N}$ & HN \\
\hline V3 & 120.085 & 8.117 & V3 & 120.441 & 8.201 \\
\hline F4 & 123.589 & 8.198 & F4 & 123.521 & 8.199 \\
\hline M5 & 122.033 & 8.122 & M5 & 121.972 & 8.137 \\
\hline K6 & 122.368 & 8.159 & K6 & 121.931 & 8.226 \\
\hline G7 & 109.767 & 8.305 & G7 & 109.770 & 8.305 \\
\hline L8 & 121.515 & 7.960 & L8 & 121.545 & 8.000 \\
\hline S9 & 116.550 & 8.218 & S9 & 115.542 & 8.148 \\
\hline K10 & 123.599 & 8.239 & K10 & 123.548 & 8.222 \\
\hline A11 & 125.089 & 8.185 & A11 & 125.339 & 8.162 \\
\hline K12 & 120.667 & 8.220 & K12 & 120.842 & 8.250 \\
\hline E13 & 121.967 & 8.358 & E13 & 122.042 & 8.346 \\
\hline G14 & 109.934 & 8.358 & G14 & 109.134 & 8.290 \\
\hline V15 & 119.920 & 7.877 & V15 & 120.084 & 7.902 \\
\hline V16 & 124.801 & 8.168 & V16 & 124.244 & 8.209 \\
\hline A17 & 128.276 & 8.334 & A17 & 127.935 & 8.311 \\
\hline A18 & 123.241 & 8.178 & A18 & 123.517 & 8.198 \\
\hline A19 & 122.680 & 8.104 & A19 & 122.662 & 8.063 \\
\hline E20 & 119.893 & 8.236 & E20 & 119.663 & 8.211 \\
\hline K21 & 122.030 & 8.230 & K21 & 121.688 & 8.176 \\
\hline $\mathrm{T} 22$ & 114.908 & 8.024 & T22 & 114.665 & 7.987 \\
\hline K23 & 123.220 & 8.231 & K23 & 123.101 & 8.230 \\
\hline Q24 & 121.809 & 8.374 & Q24 & 121.793 & 8.375 \\
\hline G25 & 110.428 & 8.394 & G25 & 110.278 & 8.377 \\
\hline V26 & 119.505 & 7.912 & V26 & 119.534 & 7.922 \\
\hline A27 & 127.316 & 8.332 & A27 & 126.889 & 8.300 \\
\hline E28 & 120.094 & 8.262 & E28 & 120.063 & 8.260 \\
\hline A29 & 124.655 & 8.174 & A29 & 124.597 & 8.172 \\
\hline A30 & 122.692 & 8.113 & A30 & 122.651 & 8.099 \\
\hline G31 & 107.716 & 8.227 & G31 & 107.625 & 8.214 \\
\hline K32 & 120.647 & 8.030 & K32 & 120.623 & 8.023 \\
\hline T33 & 115.340 & 8.123 & T33 & 115.235 & 8.114 \\
\hline K34 & 123.664 & 8.365 & K34 & 123.622 & 8.358 \\
\hline E35 & 122.065 & 8.338 & E35 & 122.076 & 8.362 \\
\hline G36 & 109.850 & 8.344 & G36 & 109.904 & 8.340 \\
\hline V37 & 119.356 & 7.806 & V37 & 119.328 & 7.811 \\
\hline L38 & 125.572 & 8.172 & L38 & 125.437 & 8.168 \\
\hline Y39 & 122.126 & 8.150 & Y39 & 122.018 & 8.140 \\
\hline V40 & 122.860 & 7.988 & V40 & 122.758 & 7.981 \\
\hline G41 & 112.012 & 7.990 & G41 & 111.968 & 7.993 \\
\hline S42 & 115.338 & 8.124 & S42 & 115.298 & 8.121 \\
\hline K43 & 123.580 & 8.363 & K43 & 123.561 & 8.363 \\
\hline T44 & 115.186 & 8.075 & T44 & 115.176 & 8.072 \\
\hline K45 & 123.664 & 8.365 & K45 & 123.574 & 8.361 \\
\hline E46 & 121.941 & 8.334 & E46 & 121.860 & 8.324 \\
\hline G47 & 109.842 & 8.339 & G47 & 109.811 & 8.340 \\
\hline V48 & 119.745 & 7.813 & V48 & 119.751 & 7.820 \\
\hline V49 & 124.841 & 8.201 & V49 & 124.699 & 8.180 \\
\hline H50 & 124.293 & 8.385 & $\mathrm{H} 50$ & 124.138 & 8.400 \\
\hline G51 & 110.060 & 8.329 & G51 & 110.071 & 8.331 \\
\hline V52 & 119.371 & 7.937 & V52 & 119.440 & 7.968 \\
\hline A53 & 127.903 & 8.382 & A53 & 127.741 & 8.379 \\
\hline
\end{tabular}




\begin{tabular}{|c|c|c|c|c|c|}
\hline T54 & 114.503 & 8.101 & T54 & 114.467 & 8.089 \\
\hline V55 & 122.963 & 8.173 & V55 & 122.933 & 8.195 \\
\hline A56 & 127.853 & 8.309 & A56 & 127.696 & 8.319 \\
\hline E57 & 120.694 & 8.269 & E57 & 120.641 & 8.260 \\
\hline V58 & 122.517 & 8.316 & V58 & 122.415 & 8.308 \\
\hline T59 & 115.566 & 8.094 & T59 & 115.491 & 8.096 \\
\hline K60 & 123.481 & 8.270 & K60 & 123.442 & 8.251 \\
\hline E61 & 121.856 & 8.358 & E61 & 122.054 & 8.354 \\
\hline Q62 & 121.571 & 8.318 & Q62 & 121.504 & 8.305 \\
\hline V63 & 121.652 & 8.174 & V63 & 121.589 & 8.167 \\
\hline T64 & 117.765 & 8.190 & T64 & 117.726 & 8.184 \\
\hline N65 & 121.446 & 8.423 & N65 & 121.446 & 8.423 \\
\hline V66 & 120.478 & 8.125 & V66 & 120.489 & 8.127 \\
\hline G67 & 112.447 & 8.449 & G67 & 112.436 & 8.450 \\
\hline G68 & 108.778 & 8.143 & G68 & 108.787 & 8.143 \\
\hline A69 & 123.738 & 8.070 & A69 & 123.744 & 8.071 \\
\hline V70 & 120.187 & 8.096 & V70 & 120.182 & 8.096 \\
\hline V71 & 124.947 & 8.263 & V71 & 124.906 & 8.261 \\
\hline $\mathrm{T} 72$ & 118.250 & 8.190 & $\mathrm{~T} 72$ & 118.238 & 8.186 \\
\hline G73 & 111.207 & 8.336 & G73 & 111.200 & 8.337 \\
\hline V74 & 119.337 & 7.980 & V74 & 119.341 & 7.984 \\
\hline $\mathrm{T} 75$ & 118.454 & 8.180 & $\mathrm{~T} 75$ & 118.406 & 8.178 \\
\hline A76 & 127.125 & 8.250 & A76 & 127.097 & 8.245 \\
\hline V77 & 119.679 & 8.014 & V77 & 119.662 & 8.010 \\
\hline A78 & 127.821 & 8.286 & A78 & 127.785 & 8.285 \\
\hline Q79 & 120.453 & 8.302 & Q79 & 120.404 & 8.298 \\
\hline K80 & 122.983 & 8.320 & K80 & 122.950 & 8.315 \\
\hline T81 & 116.552 & 8.174 & T81 & 116.558 & 8.177 \\
\hline V82 & 122.985 & 8.140 & V82 & 122.910 & 8.143 \\
\hline E83 & 125.067 & 8.469 & E83 & 125.046 & 8.466 \\
\hline G84 & 110.553 & 8.404 & G84 & 110.532 & 8.404 \\
\hline A85 & 123.918 & 8.161 & A85 & 123.918 & 8.161 \\
\hline G86 & 108.074 & 8.394 & G86 & 108.064 & 8.397 \\
\hline S87 & 115.606 & 8.066 & S87 & 115.606 & 8.066 \\
\hline I88 & 122.578 & 8.087 & I88 & 122.571 & 8.093 \\
\hline A89 & 127.805 & 8.239 & A89 & 127.682 & 8.240 \\
\hline A90 & 123.185 & 8.088 & A90 & 123.089 & 8.088 \\
\hline A91 & 123.248 & 8.179 & A91 & 123.148 & 8.174 \\
\hline T92 & 112.374 & 7.982 & T92 & 112.261 & 7.977 \\
\hline G93 & 110.585 & 8.210 & G93 & 110.520 & 8.196 \\
\hline F94 & 120.206 & 7.996 & F94 & 120.190 & 7.985 \\
\hline V95 & 123.286 & 7.960 & V95 & 123.121 & 7.948 \\
\hline K96 & 126.165 & 8.289 & K96 & 126.108 & 8.285 \\
\hline K97 & 123.653 & 8.321 & K97 & 123.627 & 8.311 \\
\hline D98 & 121.053 & 8.311 & D98 & 121.051 & 8.313 \\
\hline Q99 & 120.082 & 8.239 & Q99 & 120.056 & 8.235 \\
\hline L100 & 122.715 & 8.180 & L100 & 122.697 & 8.180 \\
\hline G101 & 109.690 & 8.382 & G101 & 109.681 & 8.384 \\
\hline K102 & 120.699 & 8.106 & K102 & 120.701 & 8.110 \\
\hline N103 & 119.948 & 8.516 & N103 & 119.948 & 8.516 \\
\hline E104 & 121.374 & 8.390 & E104 & 121.382 & 8.387 \\
\hline E105 & 122.111 & 8.318 & E105 & 122.081 & 8.320 \\
\hline G106 & 109.834 & 8.341 & G106 & 109.823 & 8.341 \\
\hline A107 & 124.898 & 8.036 & A107 & 124.898 & 8.036 \\
\hline P108 & & & P108 & & \\
\hline Q109 & 121.012 & 8.481 & Q109 & 121.005 & 8.481 \\
\hline E110 & 122.321 & 8.418 & E110 & 122.304 & 8.419 \\
\hline
\end{tabular}




\begin{tabular}{|ccc|ccc|}
\hline G111 & 110.127 & 8.385 & G111 & 110.131 & 8.388 \\
I112 & 120.008 & 7.892 & I112 & 119.990 & 7.892 \\
L113 & 126.764 & 8.295 & L113 & 126.730 & 8.293 \\
E114 & 122.103 & 8.317 & E114 & 122.078 & 8.314 \\
D115 & 121.289 & 8.257 & D115 & 121.258 & 8.258 \\
M116 & 121.825 & 8.140 & M116 & 121.824 & 8.138 \\
P117 & & & P117 & & \\
V118 & 120.577 & 8.173 & V118 & 120.560 & 8.172 \\
D119 & 125.692 & 8.397 & D119 & 125.658 & 8.396 \\
P120 & & & P120 & & \\
D121 & 119.269 & 8.299 & D121 & 119.241 & 8.297 \\
N122 & 118.992 & 8.036 & N122 & 118.992 & 8.035 \\
E123 & 121.648 & 8.295 & E123 & 121.669 & 8.299 \\
A124 & 124.286 & 8.128 & A124 & 124.256 & 8.128 \\
Y125 & 119.780 & 7.924 & Y125 & 119.750 & 7.921 \\
E126 & 123.513 & 8.056 & E126 & 123.508 & 8.055 \\
M127 & 123.654 & 8.315 & M127 & 123.627 & 8.311 \\
P128 & & & P128 & & \\
S129 & 116.611 & 8.377 & S129 & 116.582 & 8.374 \\
E130 & 123.079 & 8.477 & E130 & 123.064 & 8.476 \\
E131 & 121.966 & 8.359 & E131 & 121.946 & 8.356 \\
G132 & 109.842 & 8.345 & G132 & 109.819 & 8.342 \\
Y133 & 120.191 & 7.964 & Y133 & 120.198 & 7.965 \\
Q134 & 122.487 & 8.151 & Q134 & 122.487 & 8.152 \\
D135 & 121.618 & 8.148 & D135 & 121.597 & 8.147 \\
Y136 & 120.342 & 7.939 & Y136 & 120.369 & 7.940 \\
E137 & 125.250 & 8.153 & E137 & 125.239 & 8.149 \\
P138 & & & P138 & & \\
E139 & 121.677 & 8.444 & E139 & 121.674 & 8.444 \\
A140 & 130.758 & 7.876 & A140 & 130.758 & 7.876 \\
\hline
\end{tabular}


Table S5. Change in Chemical Shift for HN and N, occurring upon complexation of ${ }^{15} \mathrm{~N}-\mathrm{CaM}$ with alphaSynuclein

\begin{tabular}{|c|c|c|c|c|c|c|c|c|}
\hline Residue & $\begin{array}{c}\mathbf{N} \\
(\mathbf{p p m})\end{array}$ & $\begin{array}{c}\text { HN } \\
(\mathbf{p p m})\end{array}$ & Residue & $\begin{array}{c}\mathbf{N} \\
(\mathbf{p p m})\end{array}$ & $\begin{array}{c}\text { HN } \\
(\mathbf{p p m})\end{array}$ & Residue & $\begin{array}{c}\mathbf{N} \\
(\mathbf{p p m})\end{array}$ & $\begin{array}{c}\mathbf{H N} \\
(\mathbf{p p m})\end{array}$ \\
\hline A1 & 0.080 & -0.077 & M51 & -0.205 & 0.010 & S101 & 0.000 & -0.012 \\
\hline D2 & 0.142 & 0.006 & I52 & -0.041 & 0.034 & A102 & 0.133 & 0.019 \\
\hline Q3 & -0.041 & -0.036 & N53 & 0.246 & 0.034 & A103 & 0.051 & 0.013 \\
\hline L4 & 0.095 & 0.021 & E54 & -0.154 & -0.076 & E104 & 0.183 & 0.025 \\
\hline $\mathrm{T} 5$ & -0.202 & -0.004 & V55 & 1.573 & 0.023 & L105 & -0.275 & -0.067 \\
\hline E6 & -0.001 & -0.002 & D56 & -0.431 & 0.033 & R106 & 0.158 & 0.051 \\
\hline E7 & -0.002 & -0.001 & A57 & -0.286 & -0.155 & H107 & -0.197 & 0.038 \\
\hline Q8 & 0.022 & -0.019 & D58 & 0.290 & 0.044 & V108 & -0.132 & -0.006 \\
\hline I9 & -0.126 & -0.038 & G59 & 0.018 & 0.003 & M109 & -0.461 & 0.003 \\
\hline A10 & 0.029 & 0.000 & D60 & 0.037 & 0.017 & $\mathrm{~T} 110$ & 0.326 & 0.050 \\
\hline E11 & 0.061 & -0.013 & G61 & -0.193 & -0.059 & N111 & 0.119 & -0.047 \\
\hline F12 & -0.272 & -0.026 & T62 & -0.079 & 0.006 & L112 & 0.238 & 0.056 \\
\hline K13 & -0.079 & 0.004 & I63 & -0.185 & -0.108 & G113 & 0.014 & -0.016 \\
\hline E14 & 0.099 & 0.001 & D64 & 0.133 & 0.049 & E114 & -0.116 & 0.002 \\
\hline A15 & -0.194 & 0.025 & F65 & -0.056 & 0.007 & $\mathrm{~K} 115$ & 0.257 & 0.024 \\
\hline F16 & -0.157 & -0.028 & E67 & -0.013 & 0.023 & L116 & 0.198 & -0.010 \\
\hline S17 & 0.329 & 0.082 & F68 & 0.000 & -0.051 & $\mathrm{~T} 117$ & 0.101 & 0.036 \\
\hline L18 & 0.227 & -0.014 & L69 & -0.487 & -0.047 & D118 & -0.002 & 0.002 \\
\hline F19 & -0.610 & -0.081 & $\mathrm{~T} 70$ & -0.029 & 0.173 & E119 & -0.010 & -0.003 \\
\hline D20 & -0.264 & -0.025 & M71 & -0.382 & -0.012 & E120 & 0.099 & 0.001 \\
\hline K21 & -0.133 & -0.011 & M72 & 0.192 & -0.068 & V121 & -0.290 & -0.038 \\
\hline D22 & 0.125 & 0.016 & A73 & -0.559 & 0.013 & D122 & -0.135 & 0.011 \\
\hline G23 & 0.054 & -0.005 & R74 & 0.266 & -0.088 & E123 & 0.010 & 0.022 \\
\hline D24 & 0.134 & 0.051 & K75 & 0.001 & -0.011 & M124 & 0.086 & -0.021 \\
\hline G25 & -0.227 & -0.039 & M76 & -0.261 & -0.056 & $\mathrm{I} 125$ & -0.250 & -0.028 \\
\hline T26 & 0.055 & 0.043 & K77 & -0.178 & -0.068 & R126 & 0.000 & 0.023 \\
\hline $\mathrm{I} 27$ & 0.143 & 0.049 & D78 & -0.334 & -0.057 & E127 & 0.094 & -0.041 \\
\hline T28 & -0.022 & -0.039 & T79 & -0.302 & -0.063 & A128 & -0.118 & -0.021 \\
\hline T29 & -0.023 & 0.066 & D80 & 0.097 & -0.022 & D129 & 0.121 & 0.019 \\
\hline K30 & 0.117 & -0.020 & S81 & 0.071 & 0.028 & I130 & -0.008 & -0.035 \\
\hline E31 & 0.092 & 0.028 & E82 & -0.184 & -0.024 & D131 & -0.005 & 0.023 \\
\hline L32 & -0.114 & -0.016 & E83 & -0.071 & -0.028 & G132 & 0.175 & 0.052 \\
\hline G33 & 0.262 & 0.061 & E84 & -0.143 & -0.008 & D133 & 0.272 & 0.028 \\
\hline T34 & 0.093 & 0.020 & I85 & -0.321 & -0.032 & G134 & -0.101 & -0.024 \\
\hline V35 & 0.089 & 0.056 & R86 & -0.107 & 0.012 & Q135 & -0.015 & 0.005 \\
\hline M36 & 0.002 & -0.035 & E87 & -0.025 & 0.017 & V136 & -0.003 & -0.003 \\
\hline R37 & -0.193 & 0.003 & A88 & -0.193 & 0.028 & N137 & 0.097 & 0.022 \\
\hline S38 & 0.057 & 0.007 & F89 & -0.163 & -0.003 & Y138 & -0.034 & -0.037 \\
\hline L39 & -0.080 & -0.025 & R90 & 0.092 & 0.042 & E139 & 0.061 & -0.004 \\
\hline G40 & 0.014 & -0.016 & V91 & -0.123 & -0.048 & E140 & 0.051 & 0.004 \\
\hline Q41 & -0.164 & 0.003 & F92 & -0.146 & -0.073 & F141 & -0.070 & -0.063 \\
\hline N42 & 0.120 & 0.009 & D93 & -0.378 & 0.000 & V142 & -0.179 & 0.046 \\
\hline T44 & 0.099 & -0.032 & K94 & 0.003 & 0.017 & Q143 & 0.194 & 0.082 \\
\hline E45 & -0.005 & -0.003 & D95 & 0.020 & 0.016 & M144 & -0.235 & -0.053 \\
\hline A46 & -0.003 & -0.008 & G96 & 0.007 & 0.012 & M145 & 0.030 & -0.063 \\
\hline E47 & 0.001 & -0.011 & N97 & 0.070 & 0.024 & $\mathrm{~T} 146$ & -0.573 & -0.010 \\
\hline L48 & -0.390 & -0.034 & G98 & -0.055 & 0.003 & A147 & 0.205 & 0.006 \\
\hline Q49 & 0.087 & 0.020 & Y99 & 0.115 & 0.016 & K148 & 0.154 & 0.070 \\
\hline D50 & 0.092 & 0.015 & I100 & -0.016 & 0.005 & & & \\
\hline
\end{tabular}


Table S6. Change in Chemical Shift for $\mathrm{HN}$ and N, occurring upon complexation of ${ }^{15} \mathrm{~N}$-alpha-Synuclein with $\mathrm{CaM}$

\begin{tabular}{|c|c|c|c|c|c|c|c|c|}
\hline Residue & $\begin{array}{c}\mathbf{N} \\
(\mathbf{p p m})\end{array}$ & $\begin{array}{c}\text { HN } \\
(\mathbf{p p m})\end{array}$ & Residue & $\begin{array}{c}\mathbf{N} \\
(\mathbf{p p m})\end{array}$ & $\begin{array}{c}\text { HN } \\
(\mathbf{p p m})\end{array}$ & Residue & $\begin{array}{c}\mathbf{N} \\
(\mathbf{p p m})\end{array}$ & $\begin{array}{c}\text { HN } \\
(\mathbf{p p m})\end{array}$ \\
\hline V3 & 0.356 & 0.084 & V48 & 0.006 & 0.007 & G93 & -0.065 & -0.014 \\
\hline $\mathrm{F} 4$ & -0.068 & 0.001 & V49 & -0.142 & -0.021 & F94 & -0.016 & -0.011 \\
\hline M5 & -0.061 & 0.015 & $\mathrm{H} 50$ & -0.155 & 0.015 & V95 & -0.165 & -0.012 \\
\hline K6 & -0.437 & 0.067 & G51 & 0.011 & 0.002 & K96 & -0.057 & -0.004 \\
\hline G7 & 0.003 & 0.000 & V52 & 0.069 & 0.031 & K97 & -0.026 & -0.010 \\
\hline L8 & 0.030 & 0.040 & A53 & -0.162 & -0.003 & D98 & -0.002 & 0.002 \\
\hline S9 & -1.008 & -0.070 & T54 & -0.036 & -0.012 & Q99 & -0.026 & -0.004 \\
\hline K10 & -0.051 & -0.017 & V55 & -0.030 & 0.022 & L100 & -0.018 & 0.000 \\
\hline A11 & 0.250 & -0.023 & A56 & -0.157 & 0.010 & G101 & -0.009 & 0.002 \\
\hline K12 & 0.175 & 0.030 & E57 & -0.053 & -0.009 & K102 & 0.002 & 0.004 \\
\hline E13 & 0.075 & -0.012 & V58 & -0.102 & -0.008 & N103 & 0.000 & 0.000 \\
\hline G14 & -0.800 & -0.068 & T59 & -0.075 & 0.002 & E104 & 0.008 & -0.003 \\
\hline V15 & 0.164 & 0.025 & K60 & -0.039 & -0.019 & E105 & -0.030 & 0.002 \\
\hline V16 & -0.557 & 0.041 & E61 & 0.198 & -0.004 & G106 & -0.011 & 0.000 \\
\hline A17 & -0.341 & -0.023 & Q62 & -0.067 & -0.013 & A107 & 0.000 & 0.000 \\
\hline A18 & 0.276 & 0.020 & V63 & -0.063 & -0.007 & Q109 & -0.007 & 0.000 \\
\hline A19 & -0.018 & -0.041 & T64 & -0.039 & -0.006 & E110 & -0.017 & 0.001 \\
\hline E20 & -0.230 & -0.025 & N65 & 0.000 & 0.000 & G111 & 0.004 & 0.003 \\
\hline K21 & -0.342 & -0.054 & V66 & 0.011 & 0.002 & I112 & -0.018 & 0.000 \\
\hline $\mathrm{T} 22$ & -0.243 & -0.037 & G67 & -0.011 & 0.001 & L113 & -0.034 & -0.002 \\
\hline K23 & -0.119 & -0.001 & G68 & 0.009 & 0.000 & E114 & -0.025 & -0.003 \\
\hline Q24 & -0.016 & 0.001 & A69 & 0.006 & 0.001 & D115 & -0.031 & 0.001 \\
\hline G25 & -0.150 & -0.017 & V70 & -0.005 & 0.000 & M116 & -0.001 & -0.002 \\
\hline V26 & 0.029 & 0.010 & V71 & -0.041 & -0.002 & V118 & -0.017 & -0.001 \\
\hline A27 & -0.427 & -0.032 & T72 & -0.012 & -0.004 & D119 & -0.034 & -0.001 \\
\hline E28 & -0.031 & -0.002 & G73 & -0.007 & 0.001 & D121 & -0.028 & -0.002 \\
\hline A29 & -0.058 & -0.002 & V74 & 0.004 & 0.004 & N122 & 0.000 & -0.001 \\
\hline A30 & -0.041 & -0.014 & $\mathrm{~T} 75$ & -0.048 & -0.002 & E123 & 0.021 & 0.004 \\
\hline G31 & -0.091 & -0.013 & A76 & -0.028 & -0.005 & A124 & -0.030 & 0.000 \\
\hline K32 & -0.024 & -0.007 & V77 & -0.017 & -0.004 & Y125 & -0.030 & -0.003 \\
\hline T33 & -0.105 & -0.009 & A78 & -0.036 & -0.001 & E126 & -0.005 & -0.001 \\
\hline K34 & -0.042 & -0.007 & Q79 & -0.049 & -0.004 & M127 & -0.027 & -0.004 \\
\hline E35 & 0.011 & 0.024 & K80 & -0.033 & -0.005 & S129 & -0.029 & -0.003 \\
\hline G36 & 0.054 & -0.004 & T81 & 0.006 & 0.003 & E130 & -0.015 & -0.001 \\
\hline V37 & -0.028 & 0.005 & V82 & -0.075 & 0.003 & E131 & -0.020 & -0.003 \\
\hline L38 & -0.135 & -0.004 & E83 & -0.021 & -0.003 & G132 & -0.023 & -0.003 \\
\hline Y39 & -0.108 & -0.010 & G84 & -0.021 & 0.000 & Y133 & 0.007 & 0.001 \\
\hline V40 & -0.102 & -0.007 & A85 & 0.000 & 0.000 & Q134 & 0.000 & 0.001 \\
\hline G41 & -0.044 & 0.003 & G86 & -0.010 & 0.003 & D135 & -0.021 & -0.001 \\
\hline S42 & -0.040 & -0.003 & S87 & 0.000 & 0.000 & Y136 & 0.027 & 0.001 \\
\hline K43 & -0.019 & 0.000 & I88 & -0.007 & 0.006 & E137 & -0.011 & -0.004 \\
\hline T44 & -0.010 & -0.003 & A89 & -0.123 & 0.001 & E139 & -0.003 & 0.000 \\
\hline K45 & -0.090 & -0.004 & A90 & -0.096 & 0.000 & A140 & 0.000 & 0.000 \\
\hline E46 & -0.081 & -0.010 & A91 & -0.100 & -0.005 & & & \\
\hline G47 & -0.031 & 0.001 & T92 & -0.113 & -0.005 & & & \\
\hline
\end{tabular}


Table S7. PCS values experimentally determined for $\mathrm{Tb}^{3+}$ substituted ${ }^{15} \mathrm{~N}$-Calmodulin: Synuclein 1:1

\begin{tabular}{|cc|cc|}
\hline Residue & $\begin{array}{c}\text { HN } \\
\text { (ppm) }\end{array}$ & Residue & $\begin{array}{c}\text { HN } \\
\text { (ppm) }\end{array}$ \\
\hline & & & \\
D2 & 0.634 & A103 & -0.087 \\
T5 & 0.968 & R106 & -0.092 \\
E6 & 1.079 & T110 & -0.104 \\
E7 & 0.964 & K115 & -0.081 \\
Q8 & 1.005 & L116 & -0.085 \\
I & 1.610 & T117 & -0.060 \\
A10 & 1.636 & D118 & -0.063 \\
E11 & 1.204 & E119 & -0.050 \\
E12 & 1.413 & E120 & -0.061 \\
E14 & 1.510 & V121 & -0.081 \\
A15 & 1.088 & D122 & -0.065 \\
L18 & 0.806 & E123 & -0.072 \\
D22 & -0.347 & M124 & -0.075 \\
G23 & 0.698 & R126 & -0.060 \\
D24 & 0.895 & E127 & -0.081 \\
K30 & -1.666 & A128 & -0.087 \\
T34 & -1.700 & D129 & -0.086 \\
S38 & -1.251 & I130 & -0.088 \\
G40 & -1.099 & D131 & -0.081 \\
Q41 & -1.319 & G132 & -0.064 \\
N42 & -1.193 & D133 & -0.074 \\
T44 & -1.359 & G134 & -0.067 \\
E45 & -0.692 & Q135 & -0.079 \\
L48 & -2.084 & V136 & -0.104 \\
N53 & 0.193 & N137 & -0.113 \\
F92 & -0.049 & Y138 & -0.140 \\
K94 & -0.138 & E139 & -0.129 \\
D95 & -0.101 & E140 & -0.125 \\
G96 & -0.112 & F141 & -0.162 \\
N97 & -0.078 & V142 & -0.146 \\
G98 & -0.106 & Q143 & -0.159 \\
Y99 & -0.111 & M145 & -0.128 \\
I100 & -0.108 & A147 & -0.142 \\
S101 & -0.116 & K148 & -0.119 \\
A102 & -0.082 & & \\
\hline & & & \\
\hline
\end{tabular}


Table S8. PCS values experimentally determined for $\mathrm{Tm}^{3+}$ substituted ${ }^{15} \mathrm{~N}-$ Calmodulin: Synuclein 1:1

\begin{tabular}{|c|c|c|c|}
\hline Residue & $\begin{array}{c}\text { HN } \\
(\mathbf{p p m})\end{array}$ & Residue & $\begin{array}{c}\text { HN } \\
(\mathbf{p p m})\end{array}$ \\
\hline A1 & -0.401 & G98 & 0.053 \\
\hline D2 & -0.784 & Y99 & 0.082 \\
\hline Q3 & -0.535 & I100 & 0.073 \\
\hline L4 & -0.838 & S101 & 0.078 \\
\hline T5 & -0.602 & A102 & 0.060 \\
\hline E7 & -0.580 & A103 & 0.056 \\
\hline Q8 & -0.608 & E104 & 0.067 \\
\hline I9 & -0.943 & L105 & 0.074 \\
\hline A10 & -0.976 & R106 & 0.072 \\
\hline E11 & -0.709 & H107 & 0.068 \\
\hline F12 & -0.670 & V108 & 0.050 \\
\hline K13 & -1.395 & $\mathrm{~T} 110$ & 0.095 \\
\hline E14 & -0.936 & G113 & 0.080 \\
\hline A15 & -0.474 & K115 & 0.045 \\
\hline F16 & -1.498 & L116 & 0.055 \\
\hline S17 & -1.058 & $\mathrm{~T} 117$ & 0.029 \\
\hline L18 & -0.199 & D118 & 0.035 \\
\hline D22 & -0.695 & E119 & 0.042 \\
\hline G23 & -1.357 & E120 & 0.046 \\
\hline D24 & -2.320 & V121 & 0.044 \\
\hline K30 & 2.136 & D122 & 0.043 \\
\hline E31 & 1.744 & E123 & 0.039 \\
\hline L32 & 3.544 & $\mathrm{I} 125$ & 0.029 \\
\hline G33 & 2.701 & R126 & 0.049 \\
\hline T34 & 1.652 & E127 & 0.053 \\
\hline V35 & 0.914 & A128 & 0.065 \\
\hline M36 & 2.057 & D129 & 0.059 \\
\hline R37 & 1.309 & $\mathrm{I} 130$ & 0.062 \\
\hline S38 & 0.934 & D131 & 0.056 \\
\hline G40 & 0.773 & G132 & 0.052 \\
\hline Q41 & 0.640 & D133 & 0.044 \\
\hline N42 & 0.927 & G134 & 0.047 \\
\hline $\mathrm{T} 44$ & 1.087 & Q135 & 0.056 \\
\hline A46 & 0.577 & V136 & 0.061 \\
\hline E47 & 1.781 & N137 & 0.077 \\
\hline D50 & 2.041 & Y138 & 0.079 \\
\hline M51 & 4.651 & E140 & 0.087 \\
\hline R74 & 0.750 & F141 & 0.088 \\
\hline K75 & 1.287 & V142 & 0.110 \\
\hline V91 & 0.005 & M144 & 0.085 \\
\hline F92 & 0.040 & M145 & 0.095 \\
\hline D95 & 0.075 & A147 & 0.100 \\
\hline G96 & 0.062 & K148 & 0.092 \\
\hline N97 & 0.066 & & \\
\hline
\end{tabular}


Table S9. PCS values experimentally determined for Dy ${ }^{3+}$ substituted ${ }^{15} \mathrm{~N}$-Calmodulin: Synuclein 1:1

\begin{tabular}{|cc|cc|}
\hline Residue & $\begin{array}{c}\text { HN } \\
\text { (ppm) }\end{array}$ & Residue & $\begin{array}{c}\text { HN } \\
\text { (ppm) }\end{array}$ \\
\hline D2 & 1.301 & R106 & -0.082 \\
Q3 & 1.458 & K115 & -0.083 \\
L4 & 1.464 & L116 & -0.076 \\
T5 & 0.973 & T117 & -0.071 \\
E6 & 0.966 & D118 & -0.057 \\
E7 & 0.840 & E119 & -0.054 \\
I9 & 1.370 & E120 & -0.054 \\
E11 & 0.848 & V121 & -0.068 \\
F12 & 0.986 & D122 & -0.055 \\
K13 & 1.427 & E123 & -0.053 \\
E14 & 0.799 & I125 & -0.080 \\
S17 & 0.280 & E127 & -0.045 \\
D22 & -0.979 & A128 & -0.041 \\
G23 & -0.866 & D129 & -0.038 \\
D24 & -1.766 & I130 & -0.041 \\
T28 & -0.980 & D131 & -0.043 \\
K30 & 1.666 & G132 & -0.037 \\
T34 & -0.700 & D133 & -0.052 \\
T44 & -0.614 & G134 & -0.053 \\
L48 & -0.625 & Q135 & -0.055 \\
Q49 & 0.951 & V136 & -0.070 \\
D95 & -0.021 & N137 & -0.057 \\
G96 & -0.070 & Y138 & -0.055 \\
N97 & -0.059 & E139 & -0.038 \\
G98 & -0.090 & E140 & -0.032 \\
Y99 & -0.058 & F141 & -0.065 \\
I100 & -0.059 & V142 & -0.039 \\
S101 & -0.031 & Q143 & -0.018 \\
A102 & -0.066 & M145 & -0.056 \\
A103 & -0.073 & A147 & -0.004 \\
E104 & -0.070 & K148 & -0.022 \\
L105 & -0.052 & & \\
\hline & & & \\
\hline
\end{tabular}

